\title{
High Capacity Downlink Transmission with MIMO Interference Subspace Rejection in Multicellular CDMA Networks
}

\author{
Henrik Hansen \\ INRS-Télécommunications, Université du Québec, Place Bonaventure, 800 de la Gauchetière Ouest, \\ Suite 6900, Montréal, Québec, Canada H5A 1K6 \\ Email:henrik.b.hansen@ericsson.com

\section{Sofiène Affes} \\ INRS-Télécommunications, Université du Québec, Place Bonaventure, 800 de la Gauchetière Ouest, \\ Suite 6900, Montréal, Québec, Canada H5A 1 K6 \\ Email:affes@inrs-emt.uquebec.ca

\section{Paul Mermelstein} \\ INRS-Télécommunications, Université du Québec, Place Bonaventure, 800 de la Gauchetière Ouest, \\ Suite 6900, Montréal, Québec, Canada H5A 1K6 \\ Email:mermel@inrs-emt.uquebec.ca
}

Received 31 December 2002; Revised 18 September 2003

\begin{abstract}
We proposed recently a new technique for multiuser detection in CDMA networks, denoted by interference subspace rejection (ISR), and evaluated its performance on the uplink. This paper extends its application to the downlink (DL). On the DL, the information about the interference is sparse, for example, spreading factor (SF) and modulation of interferers may not be known, which makes the task much more challenging. We present three new ISR variants which require no prior knowledge of interfering users. The new solutions are applicable to MIMO systems and can accommodate any modulation, coding, SF, and connection type. We propose a new code allocation scheme denoted by DACCA which significantly reduces the complexity of our solution at the receiving mobile. We present estimates of user capacities and data rates attainable under practically reasonable conditions regarding interferences identified and suppressed in a multicellular interference-limited system. We show that the system capacity increases linearly with the number of antennas despite the existence of interference. Our new DL multiuser receiver consistently provides an Erlang capacity gain of at least $3 \mathrm{~dB}$ over the single-user detector.
\end{abstract}

Keywords and phrases: CDMA, downlink multiuser detection, interference rejection, space-time processing, code allocation, MIMO.

\section{INTRODUCTION}

Third generation wireless systems will deploy wideband CDMA (W-CDMA) $[1,2]$ access technology to achieve data transmission at variable rates. Standards [1] call for transmission rates up to $384 \mathrm{Kbps}$ for mobile users and $2 \mathrm{Mbps}$ for portable terminals. On the downlink (DL), high-speed DL packet access (HSDPA) $[3,4]$ allows for transmission rates up to about $10 \mathrm{Mbps}$ in the conventional single-input singleoutput (SISO) channel and about $20 \mathrm{Mbps}$ in the multipleinput multiple-output (MIMO) channel. It is expected that most of the traffic will be DL due to asymmetrical services like FTP and web browsing. The DL will therefore become the limiting link, and only high DL performance can give the network operator maximal revenue from advanced radionetwork technologies.

MIMO [5] and multiuser detection (MUD) $[6,7,8]$ are both very promising techniques for high capacity on the DL in wireless systems. In a noise-limited MIMO system, Shannon capacities increase linearly in SNR with the number of antennas [5] instead of logarithmically as in the SISO system. Recent studies, however, have shown that in an interferencelimited MIMO system, this linear relationship is not achieved due to the multiple-access interference (MAI) $[8]$. In $[9,10]$, it was shown that the gain in such systems is basically limited to the antenna beamforming gain at the receiver. In terms 
of system capacity, ${ }^{1}$ this means that the Erlang capacity increases linearly with the number of antennas. MUD can significantly increase the capacity further especially when interference is pronounced [11]. It is therefore of prime concern to establish a cost-effective solution that combines MIMO and MUD for optimal DL performance.

MUD is a challenging problem, not only for the uplink (UL), but even more so for the DL. On the UL, the receiving base station knows the connection characteristics of all in-cell users. The DL MUD problem is more difficult because the terminal has no knowledge of active interference, its spreading codes, SF, modulation, coding, and the connection type (packet switched or circuit switched). Furthermore, complexity considerations are more important because terminals are limited by size and price and are restricted in available power.

Most previous work was aimed at the UL (e.g., [11, $12,13,14,15,16,17,18,19,20,21])$. For the DL, blind adaptive MMSE solutions based on generalizations of singleuser detectors (SUDs) have previously been proposed for the STAR [22] receiver in [23], denoted STAR GSC, and for the RAKE [24] receiver in [25], denoted the generalized RAKE (G-RAKE). These solutions are characterized by low complexity and low risk because they impose the least change to an established technology. But they require the use of short codes and the capacity gain in a practical DL environment is limited to about $1.5-2.5 \mathrm{~dB}$ for the G-RAKE $[26,27]$ (and expectedly in the same range for STAR-GSC). In [28], a solution which offers potentially higher capacity gains is presented. Relying on the use of orthogonal variable spreading factor (OVSF) [29] codes, it probes for interference on the OVSF code tree at a high SF level in order to identify and reject codes with significant energy. This solution is complex because it rejects interference at a high SF level and is defined for rejection of in-cell interference only.

We propose a new class of MUD solutions for DL multicellular interference-limited CDMA-based MIMO systems. These new solutions are all DL variants of the previously presented interference subspace rejection (ISR) technique [30] and are therefore referred to as DLISR. The DLISR variants do not rely on prior knowledge of the interference and its properties (e.g., modulation, coding scheme, and connection type). Nor do they attempt to estimate the SF and modulation of the interference. DLISR takes advantage of a concept we denote by virtual interference rejection (VIR) combined with a new OVSF code allocation scheme denoted dynamic power-assisted channelization code allocation (DACCA). VIR reduces complexity in the receiver by attacking interference at a low SF. DACCA provides information to the terminal about the location of interference in the OVSF code-space. DLISR does not necessarily require VIR and DACCA. However, when combined with these new concepts, DLISR provides very high performance at very low

\footnotetext{
${ }^{1}$ System capacity is a measure of the total system capacity. Shannon capacity is a measure of the single link spectral efficiency.
}

complexity. As a benchmark, we consider the PIC $[16,17]$ with soft decision (PIC-SD), which can also exploit the VIR and DACCA techniques.

Performance of MUD detectors heavily relies on the distribution of interference. For instance, MUD typically offers very significant performance gains if the interference arrives from one strong source. However, if interference arrives from numerous weaker sources, MUD performance approaches SUD performance. In order to provide convincing results with regards to real-world applications, it follows that interference must be modelled realistically. We have therefore implemented a precise model as shown in Figure 1. First we establish a realistic realization of the interference using a radionetwork simulator (RNS); then this information is used for the link-level simulations to assess the BER for DLISR, PICSD, and the SUD. Repeating the cycle many times and combining the results, we arrive at system-level capacity estimates. Our link-level simulator makes assumptions very similar to those in W-CDMA standards. We do not rely on any a priori knowledge of the channel; instead we employ the STAR receiver [22] to estimate the channel. Simulations show that our new MUD consistently offers a gain of at least $3 \mathrm{~dB}$ over SUD based on maximal ratio combining (MRC) for QPSK and as much as 6.5-8.1 dB for 16 QAM. Our solution demonstrates a linear growth in Erlang capacities with the number of receiving antennas.

The main contributions of this paper are as follows. Most importantly, we propose a new solution for DL MIMO MUD in CDMA-based systems. We present the concepts of VIR and DACCA to allow for effective operation of DLISR and to reduce the complexity at the receiver significantly. Finally, we propose an RNS to generate realistic realizations of the interference in the DL MIMO system.

The paper is organized as follows. We present our linklevel signal model in Section 2. In Section 3, we derive DLISR and introduce DACCA and VIR. The RNS is presented in Section 4 . Then our system-level simulation results are presented in Section 5. Finally, our conclusions are given in Section 6 .

\section{LINK-LEVEL SIGNAL MODEL}

In this section, we discuss the link-level signal model and discuss briefly basic estimation issues. The radio-network model, which is important for the quality of our simulation results, is presented later in Section 4. Section 2.1 presents an overview of the MIMO model, Section 2.2 provides the mathematical model of the signals, and finally, Section 2.3 considers estimation of the basic parameters.

\subsection{Overview of the MIMO model}

We consider a DL MIMO CDMA system as illustrated in Figure 2. Let $(u, v)$ denote the user with index $u=1, \ldots, U_{v}$ connected to the cell with index $v=1, \ldots, N_{\text {CeLls. }}$. We define a cell as one site sector, that is, a three-sector site has three cells. $U_{v}$ is the number of users connected to the cell with index $v$ and $N_{\text {CELLS }}$ is the number of cells considered. 


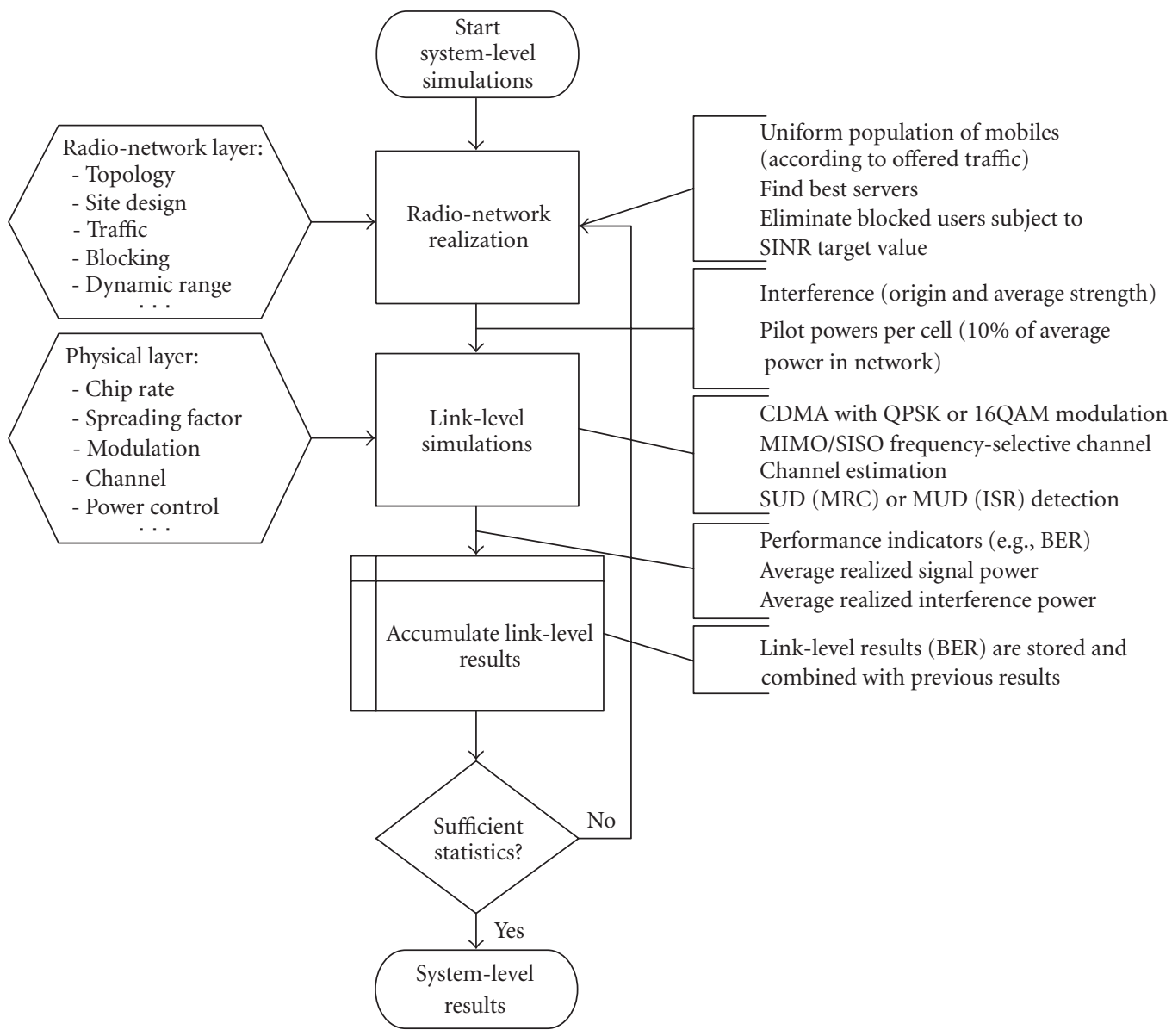

FIGURE 1: Organization of operations for radio-network and link-level simulations.

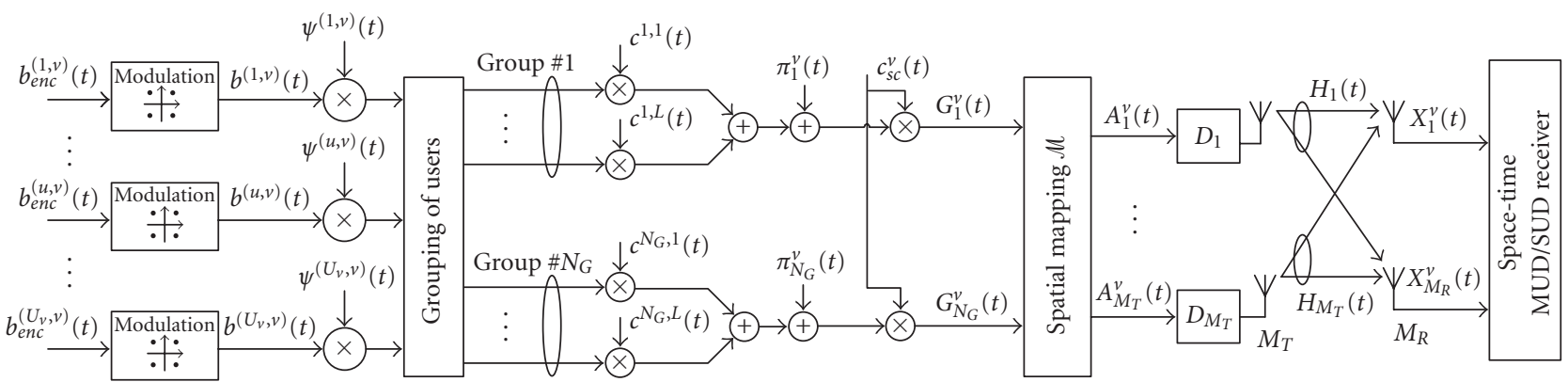

Figure 2: Block diagram of an $M_{T} \times M_{R}$ MIMO transceiver structure with emphasis on transmitter and channel.

Let $b_{\text {enc }}^{(u, v)}(t)$ represent a BPSK stream of encoded information bits. The encoded data bits are modulated according to the modulation scheme (we consider QPSK and 16-QAM in this paper) and scaled by the desired transmit amplitude $\psi^{(u, v)}(t)$. The stream of modulated channel symbols are switched to one of $N_{G}$ groups such that the user $(u, v)$ is assigned to the group $g_{(u, v)}$. The modulated symbols are then spread by a user-specific channelization code, increasing the rate by the SF, $L=T / T_{c}$, where $T$ is the time duration of one modulated symbol and $T_{c}$ is the chip duration. The channelization code is defined as $c_{\mathrm{ch}}^{(u, v)}(t)=c^{\left(g_{(u, v)}, i_{(u, v)}\right)}(t)$, where $i_{(u, v)}$ is the index to one of the codes of the group. Assignment 
of groups and channelization codes are discussed below. We add a pilot unique to each group scaled by the desired pilot amplitude, that is, $\pi_{g}^{v}(t)=\psi_{\pi}^{v}(t) c_{\pi}^{g, v}(t)$, where $\left(\psi_{\pi}^{v}(t)\right)^{2}$ is the desired pilot power and $c_{\pi}^{v, g}(t)$ is a PN code unique to the group. Finally the cell-specific scrambling code $c_{\mathrm{sc}}^{v}(t)$ is applied to yield the group-specific signal $G_{g}(t), g=1, \ldots, N_{G}$. The $N_{G}$ groups of signals, organized in the vector $G^{v}(t)=$ $\left[G_{1}^{v}(t)^{T}, \ldots, G_{N_{G}}^{v}(t)^{T}\right]^{T}$, are next spatially mapped onto $M_{T}$ antennas by the $M_{T} \times N_{G}$-dimensional matrix $\mathcal{M}$ to arrive at the $M_{T}$-dimensional signal, $A^{v}(t)=\mathcal{M} G^{v}(t) . A^{v}(t)$ is transmitted over the channel $H^{v}(t)$ and received by the mobile unit with $M_{R}$ antennas. If $\mathcal{M}$ has full rank, the groups are mapped orthogonally in space onto the transmitting antennas. Orthogonal spatial mapping is possible as long as the condition $\left(M_{T} \geq N_{G}\right)$ is satisfied. In this paper, we assume that $M_{T}=N_{G}$ and therefore the Hadamard matrix is useful. The Hadamard matrix ensures both orthogonal transmission in space and equal distribution of power between the transmitting antennas. ${ }^{2}$ If a different delay $D_{m}$ is employed at each transmitting antenna, we obtain time diversity. This may be attractive in low-diversity situations, but in a typical multipath channel possibly with multiple receive antennas, the sufficient diversity is available and extra time diversity may degrade performance because channel identification is made more difficult [31] (see also footnote 16). In our simulations, we consider multipath mostly with antenna diversity reception and therefore we have used $D_{m}=0$. Simulations (not shown herein) have demonstrated that using different antenna delays generally results in the same or slightly worse performance when multipath propagation is considered.

We now return to the concepts of grouping and channelization-code design. Channelization codes are grouped into $N_{G}$ groups with $L$ codes in each group. The purpose of grouping is to allow for user capacities beyond the SF. Each group will contain channelization codes unique to the group. Codes are correlated between groups but mutually uncorrelated within groups. The spatial mapping $\mathcal{M}$ serves to separate groups further by assigning orthogonal spatial signatures at transmission. Users are assigned a group and a channelization code pair $\left(g_{(u, v)}, i_{(u, v)}\right)$ on a first-come firstserve basis in the following order: $(g, i)=(1,1),(1,2), \ldots$, $(1, L),(2,1), \ldots,\left(N_{G}, L\right)$. Let $g_{g}$ denote the set of channelization codes in group $g$. By wise definitions of the code groups, intragroup (preferably orthogonal) as well as intergroup correlations are controlled. It is noteworthy that since the same scrambling code is used across groups, crosscorrelation properties, once set by proper choice of channelization code sets, are preserved after scrambling. As an example, we consider the following two groups of SF $=4$ channel-

\footnotetext{
${ }^{2}$ We use Hadamard matrices with a power- 2 number of transmit antennas. Otherwise, with an arbitrary number of transmit antennas, we resort to orthogonal Vandermonde-structured matrices. Current investigations suggest significant advantages due to exploitation of such spatial mapping matrices when combined with closed-loop PC and MIMO transmit diversity [31].
}

ization codes:

$$
\begin{aligned}
& \xi_{1}:\left[c^{1,1}, c^{1,2}, c^{1,3}, c^{1,4}\right]=\left[\left[\begin{array}{l}
+1 \\
+1 \\
+1 \\
+1
\end{array}\right],\left[\begin{array}{l}
+1 \\
-1 \\
+1 \\
-1
\end{array}\right],\left[\begin{array}{l}
+1 \\
+1 \\
-1 \\
-1
\end{array}\right],\left[\begin{array}{l}
+1 \\
-1 \\
-1 \\
+1
\end{array}\right]\right] ; \\
& \left.g_{2}:\left[c^{2,1}, c^{2,2}, c^{2,3}, c^{2,4}\right]=\left[\begin{array}{l}
+1 \\
-1 \\
+1 \\
+1
\end{array}\right],\left[\begin{array}{l}
+1 \\
+1 \\
+1 \\
-1
\end{array}\right],\left[\begin{array}{l}
+1 \\
-1 \\
-1 \\
-1
\end{array}\right],\left[\begin{array}{l}
+1 \\
+1 \\
-1 \\
+1
\end{array}\right]\right] .
\end{aligned}
$$

Intragroup correlations are zero for both groups and intergroup correlations are always $-6 \mathrm{~dB}$ (relatively). Using these code groups as a baseline, we can easily derive an OVSF tree for both groups (see [29]). It is easy to show that intergroup correlations reduce with higher SFs. For SF lower than four some code pairs will have nonzero correlation. Lower SFs must therefore be employed in practice with extra coordination between groups. In this example, the two code groups have been rotated by $45^{\circ}$ with respect to each other.

\subsection{Multiuser multicell downlink signal model}

We now present a mathematical formulation of the received signal. A useful diagram is shown in Figure 3. We consider the DL of a cellular CDMA system, where the mobile is equipped with an antenna array of $M_{R}$ sensors. At time $t$, the observation vector received at the antenna array of $M_{R}$ sensors at the mobile terminal can be defined as follows:

$$
X(t)=\left[\begin{array}{c}
X_{1}(t) \\
\vdots \\
X_{M_{R}}(t)
\end{array}\right]=\sum_{v=1}^{N_{\text {CELLS }}} X^{v}(t)+N(t)
$$

where

$$
X^{v}(t)=\sum_{u=1}^{U_{v}} X^{(u, v)}(t)+\sum_{g=1}^{N_{G}} X_{\pi}^{g, v}(t)
$$

is the signal arriving from the $v$ th cell, $X^{(u, v)}(t)$ is the contribution from the $(u, v)$ th user, $X_{\pi}^{g, v}(t)$ is the pilot signal of the $g$ th group of the $v$ th cell, and $N(t)$ is the thermal noise assumed to be uncorrelated additive white Gaussian noise (AWGN).

The contribution of the $(u, v)$ th user, $X^{(u, v)}(t)$, to the received signal $X(t)$ is given by

$$
X^{(u, v)}(t)=\sum_{m=1}^{M_{T}} H_{m}^{v}(t) \otimes A_{m}^{(u, v)}(t),
$$

where $H_{m}^{v}(t), m=1, \ldots, M_{T}$, is the $M_{R}$-dimensional channel vector from the $m$ th transmitting antenna to the receiving antenna array with $M_{R}$ sensors, and $A_{m}^{(u, v)}(t), m=1, \ldots, M_{T}$, is the contribution of the $(u, v)$ th user to the signal transmitted at the $m$ th antenna. Each dimension corresponds to one transmit antenna. The total transmitted signal arriving from 


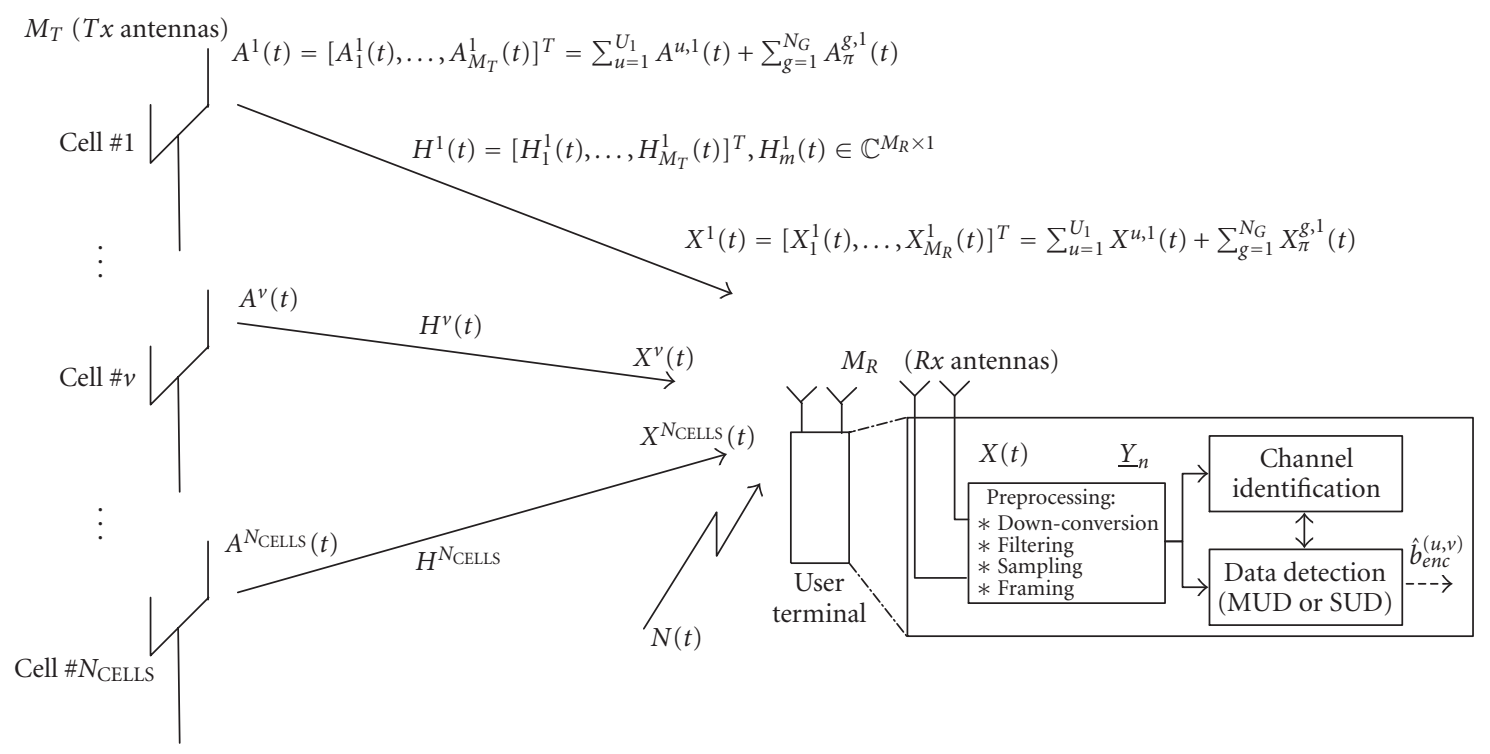

Figure 3: Network-level signal diagram.

the $(u, v)$ th user is defined as follows:

$$
A^{(u, v)}(t)=\left[\begin{array}{c}
A_{1}^{(u, v)}(t) \\
\vdots \\
A_{M_{T}}^{(u, v)}(t)
\end{array}\right]=\mathcal{M} G^{(u, v)}(t)
$$

with

$$
\begin{aligned}
G^{(u, v)}(t) & =\left[\begin{array}{c}
\vdots \\
G_{g}^{(u, v)}(t) \\
\vdots
\end{array}\right], \\
G_{g}^{(u, v)}(t) & = \begin{cases}\psi^{u, v}(t) c^{u, v}(t) b^{(u, v)}(t) & \text { if }(u, v) \in g_{g}, \\
0 & \text { if }(u, v) \notin g_{g},\end{cases}
\end{aligned}
$$

where $\left(\psi^{(u, v)}(t)\right)^{2}$ is the power, $c^{(u, v)}(t)=c_{c h}^{(u, v)}(t) c_{\mathrm{sc}}^{(u, v)}(t)$ is the spreading code (channelization code + scrambling code), and $b^{(u, v)}(t)$ denotes the modulated symbols. For lack of space, we do not detail the contribution of the pilots to the received signal, but it follows the pattern of (4), (5), and (6) by replacing $X^{(u, v)}(t)$ by $X_{\pi}^{(u, v)}(t), A^{(u, v)}(t)$ by $A_{\pi}^{(u, v)}(t)$, and $G^{(u, v)}(t)$ by $G_{\pi}^{(u, v)}(t)$, respectively.

We adopt the common assumption that the channel response can be modeled as a tapped delay line with Rayleighfaded tap gains [32]. The $M_{R}$-dimensional channel response vector from the transmitting cell to the mobile unit with $M_{R}$ antenna elements is therefore given as follows:

$$
H^{v}(t)=\left[\begin{array}{c}
\vdots \\
H_{m}^{v}(t) \\
\vdots
\end{array}\right], \quad m=1, \ldots, M_{T}
$$

with

$H_{m}^{v}(t)=L_{\mathrm{LOSS}} \sum_{p=1}^{P} h_{m, p}^{v}(t) \varepsilon_{p}^{v}(t) \delta\left(t-\tau_{p}^{v}-D_{m}\right), \quad m=1, \ldots, M_{T}$

where $\delta(t)$ is the Dirac delta function, $\tau_{p}^{\nu}(t) \in[0, T)$ are the multipath time delays for $p=1, \ldots, P$. Note that the physical path delays are the same for all receiving antennas but delay differences may optionally be imposed at transmission. $h_{m, p}^{v}(t)=\left[h_{1, m, p}^{v}(t), \ldots, h_{M_{R}, m, p}^{v}(t)\right]^{T}$ is the unit-norm propagation vector, $\varepsilon_{p}^{v}(t)^{2}, p=1, \ldots, P$, are the power fractions along each path such that $\sum_{p=1}^{P} \varepsilon_{p}^{v}(t)^{2} \doteq 1, D_{m}$ is an additional transmit delay associated with each transmit antenna, and $L_{\mathrm{LOSS}}$ is the path loss. In practice, $L_{\mathrm{LOSS}}$ is largely compensated by power control and we therefore fix it to unity in what follows. Note that this implies that the expected gain of $H_{m}^{v}(t)$ is one (by definition).

At reception, the $M_{R}$-dimensional received signal is first filtered by the pulse-matched filter, then sampled and framed into observation vectors containing $Q$ consecutive symbols of the desired user (the signal is first down converted in reality). We define the preprocessing step through the function $\mathcal{P}, \underline{V}=\mathcal{P}(U(t), n): \mathcal{C}^{M_{R} \times 1} \rightarrow \mathcal{C}^{M_{R}\left(Q L+L_{\Delta}\right) \times 1}$ as follows (see [30] for more details):

$$
\begin{aligned}
U^{\phi}(t)= & \frac{1}{T_{c}} \int U\left(t+t^{\prime}\right) \phi\left(t^{\prime}\right) d t^{\prime} \\
\underline{V}= & {\left[U^{\phi}\left(n T+a T_{c}\right)^{T}, U^{\phi}\left(n T+(a+1) T_{c}\right)^{T}, \ldots,\right.} \\
& \left.U^{\phi}\left(n T+\left(a+Q L+L_{\Delta}-1\right) T_{c}\right)^{T}\right]^{T}
\end{aligned}
$$

where $L_{\Delta}$ is an extra margin to account for the delay spread, 
$\phi(t)$ is the square-root raised-cosine shaping pulse, and $a$ is an offset that guarantees that the targeted symbols $n Q+k$, $k=0, \ldots, Q-1$, occur within the duration of the observation frame. Without loss of generality, we set $a=0$ in what follows. With this definition, we can now define the preprocessed observation as

$$
\begin{aligned}
\underline{Y}_{n}= & \sum_{v=1}^{N_{\text {CELLS }}} \sum_{u=1}^{U_{v}} \psi_{n}^{(u, v)} \underline{Y}_{n}^{(u, v)} \\
& +\sum_{v=1}^{N_{\text {CELLS }}} \sum_{g=1}^{N_{G}} \psi_{\pi, n}^{g, v} \underline{Y}_{\pi, n}^{g, v}+\underline{N}_{n}^{\mathrm{pwn}}
\end{aligned}
$$

where $\underline{Y}_{n}=\mathcal{P}(X(t), n), \underline{Y}_{n}^{(u, v)}=\mathcal{P}\left(X^{(u, v)}(t) / \psi^{(u, v)}(t), n\right)$, $\underline{Y}_{\pi, n}^{g, v}=\mathcal{P}\left(X_{\pi}^{g, v}(t) / \psi_{\pi}^{g, v}(t), n\right), \underline{N}_{n}^{\mathrm{pwn}}=\mathscr{P}(N(t), n)$, and $\psi_{n}^{(u, v)}$ $=\psi^{(u, v)}(n Q T) . \underline{Y}_{n}^{(u, v)}$ is to be understood as the contribution of the $(u, v)$ th user to the $n$th observation. It is useful to decompose its contributions as follows:

$$
\psi_{n}^{(u, v)} \underline{Y}_{n}^{(u, v)}=\psi_{n}^{(u, v)} \sum_{k^{\prime}} b_{n Q+k^{\prime}}^{(u, v)} \underline{Y}_{k^{\prime}, n}^{(u, v)}
$$

and $\underline{Y}_{k^{\prime}, n}^{(u, v)}$ is to be understood as the signature of the $n Q+$ $k^{\prime}$ th symbol. We next define the user $\left(d, v_{d}\right)$ as the desired user $\left(v_{d}\right.$ denotes the best server of user $d$ ) and let $g_{d}$ denote the group to which the user is assigned. We now isolate the desired signal and pilot in (10) from intersymbol interference (ISI) and in-cell/out-cell MAI as follows:

$$
\begin{aligned}
\underline{Y}_{n}= & \underbrace{b_{n Q+k}^{\left(d, v_{d}\right)} \psi_{n}^{\left(d, v_{d}\right)} \underline{Y}_{k, n}^{\left(d, v_{d}\right)}}_{\text {desired signal }}+\underbrace{\psi_{\pi, n}^{g_{d}, v_{d}} \underline{Y}_{\pi, n}^{g_{d}, v_{d}}}_{\text {desired pilot }}+\underbrace{\underline{I}_{\pi, n}^{\left(d, v_{d}\right)}}_{\text {pilot interference }} \\
& +\underbrace{\underline{I}_{\mathrm{ISI}, k, n}^{\left(d, v_{d}\right)}}_{\text {ISI }}+\underbrace{\sum_{u=1, u \neq d}^{U_{v_{d}}} \underline{I}_{n}^{\left(u, v_{d}\right)}}_{\text {in-cell MAI }}+\underbrace{\sum_{v=1, v \neq v_{d}}^{N_{\text {CELLS }}} \sum_{u=1}^{U_{v}} \underline{I}_{n}^{(u, v)}}_{\text {out-cell MAI }}+\underbrace{N_{n}^{\mathrm{pwd}}}_{\text {AWGN }},
\end{aligned}
$$

where with reference to (11), we have

$$
\begin{aligned}
\underline{I}_{\mathrm{ISI}, k, n}^{\left(d, v_{d}\right)} & =\sum_{k^{\prime} \neq k} \psi_{n}^{(u, v)} b_{n Q+k^{\prime}}^{(u, v)} \underline{Y}_{k^{\prime}, n}^{\left(d, v_{d}\right)}, \\
\underline{I}_{n}^{(u, v)} & =\sum_{k^{\prime}} \psi_{n}^{(u, v)} b_{n Q+k^{\prime}}^{(u, v)} \underline{Y}_{k^{\prime}, n}^{(u, v)}, \\
\underline{I}_{\pi, n}^{d, v_{d}} & =\sum_{v=1}^{N_{\mathrm{CELLS}}} \sum_{g=1}^{N_{G}} \psi_{\pi, n}^{g, v} \underline{Y}_{\pi, n}^{g, v}-\psi_{\pi, n}^{g_{d}, v_{d}} \underline{Y}_{\pi, n}^{g_{d}, v_{d}} .
\end{aligned}
$$

\subsection{Basic parameter estimation principles}

In our simulations, we estimate every parameter as needed with no prior information assumed known to the receiver. To estimate the multipath delays and the multipath gains, we employ a variant of the STAR receiver [22] as discussed in Section 2.3.1. MRC data detection (used by the SUD considered herein), power estimation, and signal-to-interferenceplus-noise ratio (SINR) estimation for PC are then discussed in Sections 2.3.2, 2.3.3, and 2.3.4, respectively.

\subsubsection{STAR: the spatio-temporal array-receiver}

We employ a variant of the STAR receiver [22] which mainly differs in the despreading operation. Instead of using the code of the desired user for despreading, we employ a more generalized code for despreading. We consider multicodes to represent one cooperative code for despreading, which is a combination of concatenating codes in time (i.e., consecutive symbols by data remodulation) and combining over channels. For the channel of the desired user, we combine the pilot code with the data remodulated spreading code over $Q$ consecutive symbols. For other channels, we employ only the pilot for channel identification with STAR.

\subsubsection{MRC beamforming and data detection}

The signal component $s_{n Q+k}^{(u, v)}=\psi_{n}^{(u, v)} b_{n Q+k}^{(u, v)}$ contains sufficient statistics for the estimation of both data and power. The signal component can be estimated by MRC which is optimal in white noise. With reference to (12), the MRC combiner for the $k^{\prime}$ th symbol of user $(u, v)$ is as follows:

$$
\underline{W}_{\mathrm{MRC}, k^{\prime}, n}^{(u, v)}=\frac{\underline{Y}_{k^{\prime}, n}^{(u, v)}}{\left\|\underline{Y}_{k^{\prime}, n}^{(u, v)}\right\|^{2}}, \quad k^{\prime}=0, \ldots, Q-1,
$$

and then the signal component is estimated as

$$
\hat{s}_{n Q+k^{\prime}}^{(u, v)}=\underline{W}_{\mathrm{MRC}, k^{\prime}, n}^{(u, v), H} \underline{Y}_{n} .
$$

A beamformer for the pilots can be defined accordingly. Note that we use the term beamformer because $\underline{W}_{\mathrm{MRC}, k^{\prime}, n}$ works in both space and time. The transmitted symbol is estimated as the symbol in the signal constellation which is the closest to $\hat{b}_{n Q+k}^{(u, v)}=\hat{s}_{n Q+k}^{(u, v)} / \hat{\psi}_{n}^{(u, v)}$, where $\hat{\psi}_{n}^{(u, v)}$ is the estimated power (Section 2.3.3).

\subsubsection{Power estimation}

We consider two different power estimators. The first estimator first estimates the amplitude

$$
\begin{aligned}
& \hat{\psi}_{n}^{(u, v)} \\
& \quad=\alpha \hat{\psi}_{n-1}^{(u, v)}+(1-\alpha) \frac{1}{Q} \mathfrak{R}\left\{\sum_{k^{\prime}=0}^{Q-1}\left(\hat{b}_{n Q+k^{\prime}}^{(u, v)}\right)^{H} \hat{s}_{n Q+k^{\prime}}^{(u, v)} /\left|\hat{b}_{n Q+k^{\prime}}^{(u, v)}\right|\right\},
\end{aligned}
$$

where $\alpha$ is a forgetting factor. The power estimate is then found by squaring the amplitude estimate. The second estimator estimates the power directly:

$$
\left(\hat{\psi}_{n}^{(u, v)}\right)^{2}=\alpha\left(\hat{\psi}_{n-1}^{(u, v)}\right)^{2}+(1-\alpha) \frac{1}{Q} \sum_{k^{\prime}=0}^{Q-1}\left|\hat{s}_{n Q+k^{\prime}}^{(u, v)}\right|^{2} .
$$

The latter is biased because it effectively estimates the combined signal and interference noise power. The estimator in (16) has less bias and is more accurate because the filtering appears before the squaring; but it requires that the decision feedback (DF) is decent. The estimator of (17) is useful to 
TABLE 1: Definition of the constraint matrix of each mode. (Each generic column $\underline{\hat{C}}_{j, n}$ is normalized to one.)

\begin{tabular}{llr}
\hline ISR mode & $\hat{\mathrm{C}}_{n}=\left[\ldots, \underline{\hat{C}}_{j, n}, \ldots\right]$ & $N_{c}$ (number of constraints) \\
\hline Hypotheses (H) (constraint/symbol/interferer) & {$\left[\ldots, \underline{\hat{Y}}_{k, n}^{i}, \ldots\right]$} & $(Q+2) N I$ \\
Realizations (R) (constraint/interferer) & {$\left[\ldots, \sum_{k=-1}^{Q} \hat{b}^{i}{ }_{n Q+k} \underline{\underline{Y}}_{k, n}^{i}, \ldots\right]$} & $N I$ \\
Total realization (TR) (constraint/total MAI) & {$\left[\sum_{i=1}^{N I} \hat{\psi}_{n}^{i} \sum_{k=-1}^{Q} \hat{b}^{i}{ }_{n Q+k} \underline{\hat{Y}}_{k, n}^{i}\right]$} & 1 \\
\hline
\end{tabular}

estimate the power of the interference (where decision feedback is difficult), whereas the estimator of (16) is used for the desired pilot and data signal.

\subsubsection{SINR estimation}

The PC command is determined by comparing the SINR estimate at the receiver with the target SINR. We use the following estimator for the SINR:

$$
\hat{\gamma}_{n}^{\left(d, v_{d}\right)}=\left(\frac{\hat{\psi}_{n}^{\left(d, v_{d}\right)}}{\hat{\sigma}_{n}^{\left(d, v_{d}\right)}}\right)^{2},
$$

where $\hat{\psi}_{n}^{\left(d, v_{d}\right)}$ results from (16) and $\hat{\sigma}_{n}^{\left(d, v_{d}\right)}$ is an estimator for the postcombined noise, which is obtained by estimating the total received power (of all users) after combining and then subtracting the estimated power of the desired user.

\section{DOWNLINK INTERFERENCE SUBSPACE REJECTION}

Our main contribution is a new efficient and cost-effective MUD solution for DL MIMO, DLISR. DLISR is based on ISR previously presented for UL systems [30]. It incorporates new variants of ISR modes which are specially suited for the more problematic DL case. In particular, DLISR employs VIR, which involves rejection of virtual users instead of physical users. VIR has many benefits especially when it is combined with DACCA. Neither VIR nor DACCA are indispensable for DLISR; however, capacity gains and especially complexity reductions are achieved when combined. We next review ISR in Section 3.1. Then we define DACCA and VIR and introduce DLISR. Finally, we discuss the attractive complexity features of our new solutions.

\subsection{Review of ISR}

In this section, we provide an overview of ISR. For a more complete picture, see [30]. The basic ISR recipe is to form a constraint matrix $\hat{\mathbf{C}}$ with a column span which spans the estimated interference subspace. In a second step, the observation is mapped away from the interference subspace spanned by $\hat{\mathbf{C}}$ by constrained spatio-temporal projection; thereby, MAI and ISI are reduced significantly. The desired signal can then be estimated by conventional beamforming, ${ }^{3}$ for example, MRC.

\footnotetext{
${ }^{3}$ We use the term beamforming because our solution works in space and time. However, the term filter-combiner could equally well be used.
}

The projection and combining steps can also be carried out in a single beamforming step. The ISR beamformer $W_{k, n}^{\left(d, v_{d}\right)}, k=0, \ldots, Q-1$, is defined by

$$
\begin{gathered}
\mathbf{Q}_{n}=\left(\hat{\mathbf{C}}_{n}^{H} \hat{\mathbf{C}}_{n}\right)^{-1}, \\
\boldsymbol{\Pi}_{n}=\mathbf{I}_{N_{T}}-\hat{\mathbf{C}}_{n} \mathbf{Q}_{n} \hat{\mathbf{C}}_{n}^{H}, \\
\underline{W_{k, n}^{\left(d, v_{d}\right)}}=\frac{\boldsymbol{\Pi}_{n} \underline{\hat{Y}}_{k, n}^{\left(d, v_{d}\right)}}{\underline{\hat{Y}}_{k, n}^{\left(d, v_{d}\right)^{H}} \boldsymbol{\Pi}_{n} \underline{\hat{Y}}_{k, n}^{\left(d, v_{d}\right)}},
\end{gathered}
$$

where $\mathbf{I}_{N_{T}}$ denotes an $N_{T} \times N_{T}$ identity matrix, and $N_{T}=$ $M_{R}\left(Q L+L_{\Delta}\right)$ is the total space-time dimension. First, we form the projector $\Pi_{n}$ orthogonal to the constraint matrix $\hat{\mathbf{C}}_{n}$. Second, we project the estimated response vector $\underline{\hat{Y}}_{k, n}^{\left(d, v_{d}\right)}$ and normalize it to yield the ISR beamformer $\underline{W}_{k, n}^{\left(d, v_{d}\right)}$.

\subsubsection{ISR modes}

The ISR modes differ in the construction of the constraint matrix. Table 1 defines the constraint matrix of each mode when considering only MAI rejection and a pedagogical illustration is provided in Figure 4 which links the modes to the composition of the constraint matrix. In the table, NI denotes the number of interfering signals to be rejected, and $i$ is the index to a subset of MAI signals which we strive to reject. Note that for simplicity, Table 1 defines the composition of the constraint matrix when only MAI is rejected, but it is easily generalized to also incorporate ISI rejection by adding columns of the estimated ISI. Of the modes previously presented, three merit discussion here.

In the ISR-hypothesis mode (ISR-H), every symbol signature ${ }^{4}$ of the selected interfering users is rejected individually. This mode does not require DF. If the channel is known, selected interfering users can be rejected perfectly but the white noise is enhanced. ISR-H was found to perform poorly on the UL because of the large noise enhancement associated with the many constraints [30]. Its application to the $\mathrm{DL}$, however, is more appealing due to the adverse near-far situations there as we will witness later.

In the ISR-realizations mode (ISR-R), we do not form a null constraint for each symbol signature of each interfering user. Instead, we reconstruct the sequence of symbols over

\footnotetext{
4"Symbol signature" is understood as the unmodulated symbol.
} 


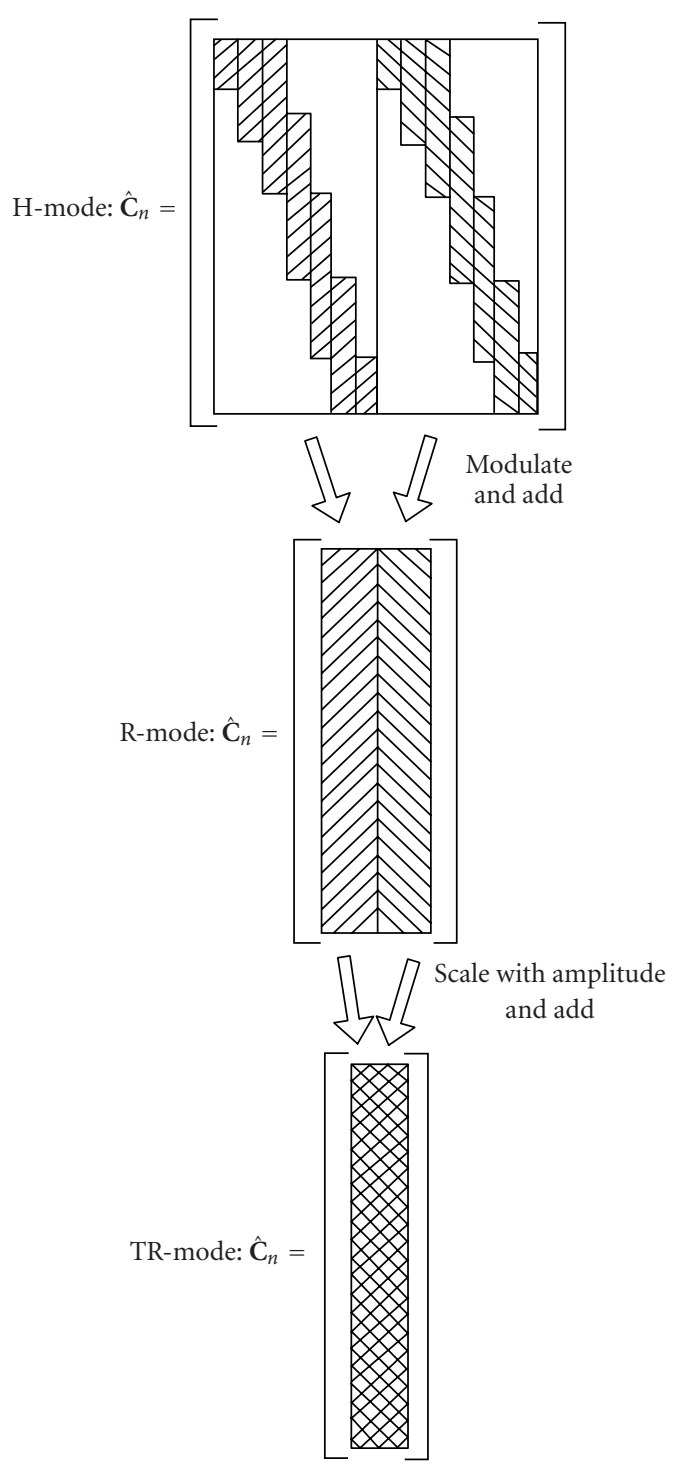

FIGURE 4: Relation between H, R, and TR modes can be illustrated from the composition of the constraint matrix.

the duration of the observation frame. The $\mathrm{R}$ mode therefore requires DF. These decisions are obtained from MRCbased decisions (Section 2.3.2). The number of constraints is reduced with ISR-R giving less white noise enhancement at the cost of reduced near-far resistance.

In the ISR-total realization (ISR-TR) mode, we reconstruct interference using DF as in the R mode, then we add the reconstructed interfering users scaled by their estimated amplitudes to form one constraint only. ISR-TR, in addition to DF, also requires power estimates (Section 2.3.3). The TR mode has negligible white noise enhancement but also the worst near-far resistance.

Before we introduce the proposed application of ISR to the DL (DLISR) in Section 3.4, we will present DACCA and VIR in Sections 3.2 and 3.3, respectively.

\subsection{DACCA}

We propose a strategy for channelization code allocation of user data channels at the base station, which we denote by DACCA. With DACCA, the base station dynamically reassigns channelization codes to the users at a low rate with the aim of concentrating energy in the left-hand side of the OVSF tree. We propose a simple metric for code assignment as the product between each user's output power and SF, denoted by the power-SF product (PSFP) in the following. ${ }^{5}$ DACCA is illustrated in Figure 5a. The aim is to fill the OVSF tree from left to right subject to the PSFP of users. The desired outcome is a concentration of power at the left-hand side of the OVSF tree. Figure 6 shows the probabilistic origin of the interference for a random mobile in a network. The distributions were obtained with the aid of the RNS to be presented in Section 4 and corresponds to a soft-blocking rate (SBR) (see Section 4.2) of $20 \%$, processing gain (PG) of 16 , and an offered traffic of $T_{\mathrm{OFF}}=4 \mathrm{Erl}$. In this paper, the PG is defined as the SF, $L$, multiplied by the number of receive antennas, that is, $\mathrm{PG}=M_{R} L$. Otherwise, the assumptions specified in Section 5.2.1 apply. We observe that most of the interference is generated by just a few users. For example, $30 \%$ of the total interference arrives from the strongest in-cell interferer and the sum of only two interferers accounts for almost half the interference. With DACCA, therefore, most of the interference power can be concentrated in a relatively small portion of the OVSF code space. It is the pronounced near-far situations on the DL which make DACCA especially interesting.

Dynamic code assignment and reassignment strategies have previously been considered in $[33,34]$. The goal in previous works was to reduce code blocking and limit the code reassignment rate. Instead, the purpose of DACCA is to provide the mobile with a priori knowledge on where to look for interference and at the same time concentrating the interference energy in a small portion of the OVSF tree. DACCA shares some similarities with the strategy denoted "leftmost" in [34], namely, users are assigned to the leftmost available code in the OVSF tree. DACCA imposes additional restrictions because it both strives to assign the leftmost codes and at the same time to achieve the best possible concentration of power at the left-hand side of the OVSF tree. Therefore, DACCA will exacerbate the probability of code blocking and more frequent code reassignments must be performed by UTRAN (UMTS terrestrial radio access network). The need for frequent reassignment is satisfied by reassigning codes at a low rate of $75 \mathrm{~Hz}$ in our simulations. Regarding code blocking, previous results [34] indicate that a load (i.e., number of OVSF codes in use divided by the SF) of $50 \%$ yields a code-blocking rate less than $1 \%$. Comparing this blocking with the loads we can achieve (see Section 5) and the SBR on the air interface, it is reasonable to deem code blocking

\footnotetext{
${ }^{5}$ In practice, the assignment rule should be more complex because not all SFs are equally probable and because assigned codes mutually preclude each other; for example, assignment of a high SF code blocks any parents of that code to be assigned. This issue is irrelevant for this work because we consider only one SF for all users in our simulations.
} 

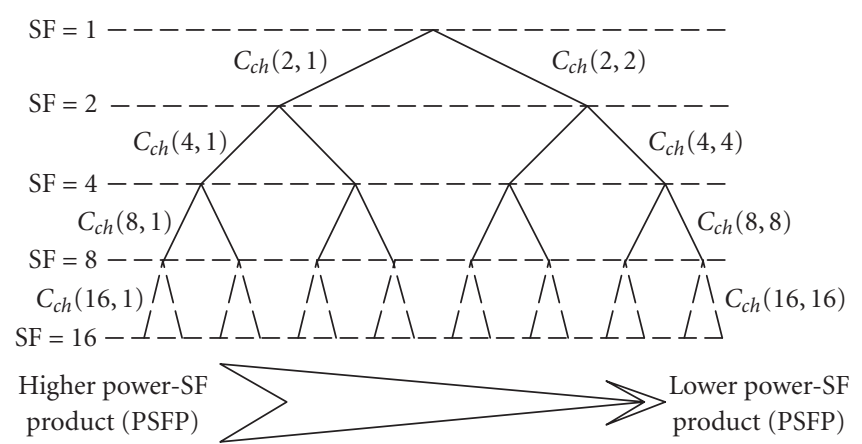

(a)

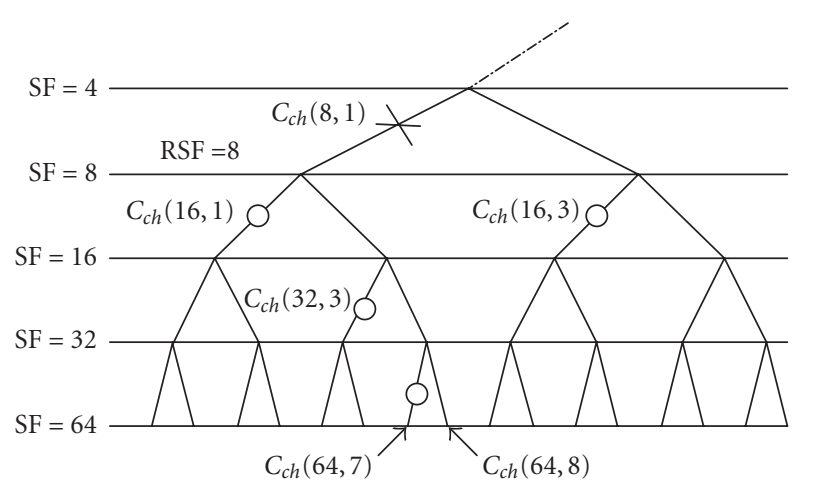

(b)

FIGURE 5: DACCA and VIR illustrated. (a) In DACCA, users are assigned channelization codes according to their PSFP. (b) Interference rejection is aimed at a low SF when VIR is employed.

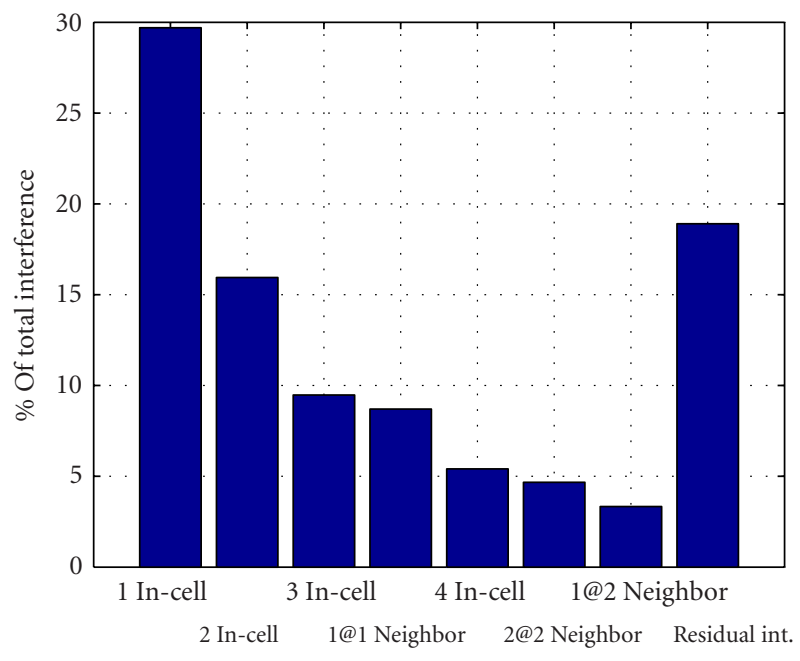

FIGURE 6: Relative power of interferers arriving from different sources. 1 In-cell is the strongest in-cell interferer, 1@1 neighbor is the strongest interference from first-tier neighbors.

to be a minor drawback of DACCA. Note that DACCA does not conflict with $3 \mathrm{G}$ standards because channelization codes can be allocated almost freely by UTRAN. Only the primary $\mathrm{CPICH}$ and the primary $\mathrm{CCPCH}$ have predefined channelization codes [29].

\subsection{Virtual interference rejection}

VIR involves rejection of interference targeting a channelization code with low SF (rejection SF (RSF)) although no physical users may be assigned this code. VIR is particularly interesting in the context of OVSF trees [29]. The idea is to target one or more virtual channelization codes with low RSF $L_{R}$ and reject these codes as if they were physical users. The advantage is that any offspring (in the OVSF tree) from the rejected virtual code is also rejected; therefore, multiple in- terfering users are rejected, targeting only a few virtual channelization codes.

It is noteworthy that VIR targets the channelization codes. In practice, the channelization codes are repeated at the rate $L_{R} T_{c}$, scrambled by the scrambling code and filtered by the channel response. A mathematical formulation of VIR is provided in [35]; here we will provide an example of VIR. Consider the segment of an OVSF tree starting at an SF of 8 shown in Figure $5 \mathrm{~b}$. Codes that are circled are in active use. Consider the virtual channelization code $c_{c h}(8,1)$, marked with an "x." We reconstruct all required segments ${ }^{6}$ of $c_{c h}(8,1)$, apply the appropriate scrambling code, and filter them by the estimated channel response. Then we reject all reconstructed segments. It then follows that all descendants are rejected irrespective of their SF and modulation; that is, the interferer with SF $=16$ assigned to code $c_{c h}(16,1)$, the code with $\mathrm{SF}=32$ assigned to $c_{c h}(32,3)$, and the one with an SF of $L=64$ assigned to $c_{c h}(64,7)$, respectively, are all rejected. The code $c_{c h}(64,8)$ is rejected although it is not active and the code $c_{c h}(16,3)$ is active but not rejected. Preferably, codes that are not active should not be rejected.

When VIR is combined with DACCA, cancelling the leftmost code at any RSF ideally causes the highest possible fraction of the interference to be rejected. The efficiency of VIR is, therefore, enhanced when DACCA is used. If DACCA is not employed, the RSF must be higher to minimize the number of rejected inactive codes. This will increase complexity significantly (see Section 3.5) and possibly degrade performance.

An idea similar to VIR was considered in [28]; however, the targeted SFs were very high SFs instead of very low SFs like in VIR. The idea there is that one interferer at a low SF is equivalent to numerous high SF virtual users. With VIR, the

\footnotetext{
${ }^{6}$ Required segments means those segments which will have contributions within the current observation frame. If the delay spread is low, there are approximately $Q L / L_{R}+1$ contributing segments per targeted virtual code (including two edge symbols).
} 


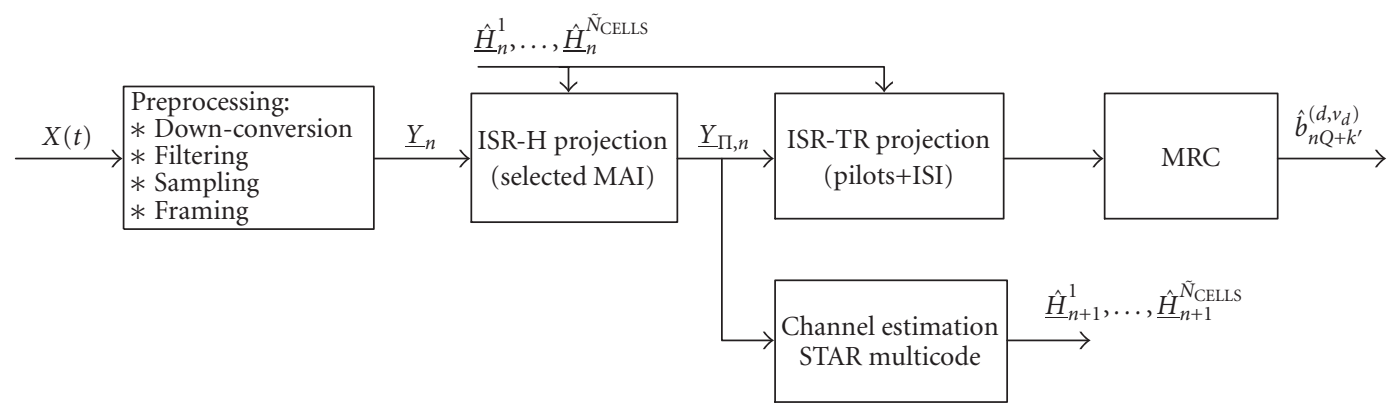

Figure 7: Proposed DLISR receiver structure. $\tilde{N}_{\text {CELLS }} \leq N_{\text {CELLS }}$ is the number of (virtual) interferers selected for rejection.

TABLE 2: Important characteristics of new ISR variants for DL MIMO.

\begin{tabular}{lcccccc}
\hline $\begin{array}{l}\text { Feature } \\
\text { strategy }\end{array}$ & $\begin{array}{c}\text { Requires } \\
\text { DACCA? }\end{array}$ & $\begin{array}{c}\text { Knows int. } \\
\text { codes? }\end{array}$ & $\begin{array}{c}\text { Knows } \\
\text { int. SF? }\end{array}$ & $\begin{array}{c}\text { Knows int. } \\
\text { modulation? }\end{array}$ & $\begin{array}{c}\text { Applicable to } \\
\text { PS/CS int.? }\end{array}$ & $\begin{array}{c}\text { Knows int. } \\
\text { coding? }\end{array}$ \\
\hline ISR-H-FC & Yes & No & No & No & Yes & No \\
ISR-H-BC & No $^{1,2}$ & No & No & No & Yes $^{3}$ & No \\
ISR-R-SD & $\mathrm{No}^{1,2}$ & No & No & No & Yes $^{3}$ & No \\
PIC-SD & No $^{1,2}$ & No & No & No & Yes $^{3}$ & No \\
MRC & No & No & No & No & Yes & No \\
\hline
\end{tabular}

${ }^{1}$ Performance gain with DACCA.

${ }^{2}$ Complexity reduction with DACCA.

${ }^{3}$ Possible performance penalty for PS.

idea is opposite: one low SF code constitutes many interferers assigned to physical OVSF codes of higher SFs.

\subsection{DLISR}

Compared to the UL, DL MUD is characterized by a lack of information regarding the interference. A mobile generally has no knowledge of the interfering users' codes, modulation, connection type, and coding. This information is only available for the pilots and the desired signal. Therefore, the interference rejection is conveniently split into two steps: in the first step, we remove the MAI and in the second step, we remove the ISI and the pilots as shown in Figure 7. The TR mode has shown excellent performance in [30] with the lowest possible complexity. Therefore, the TR mode is well suited for application in the second step regardless of the solution applied in the first step. For lack of space, we disregard further details and focus on the more important first step in the following. Improved near-far resistant channel estimation [36] may be achieved by using the near-far resistant observation $\underline{Y}_{\Pi, n}=\Pi_{n} \underline{Y}_{n}$ (see (20)) offered as an intermediate step according to Figure 7 . It is therefore natural to use $\underline{Y}_{\Pi, n}$ for the purpose of channel identification because it is offered without additional complexity. In the following, we present three variants of DLISR. Two variants based on ISR-H and are denoted by DLISR-H with fixed constraints (DLISR-HFC) and DLISR-H with best constraints (DLISR-H-BC), respectively. The final variant is based on the $\mathrm{R}$ mode with soft decision and is denoted by DLISR-R-SD. For the purpose of comparison, we also consider the PIC-SD. Important properties of the DLISR variants, PIC-SD, and MRC are summarized in Table 2.

\subsubsection{DLISR-H-FC}

DLISR-H-FC is the simplest of all variants. The idea is to blindly reject the same OVSF code subspace according to a fixed strategy. Obviously, this mode is relevant only when DACCA is employed.

Whenever a virtual-user code is rejected, white noise is enhanced. It can be shown that if the spreading is real, the noise enhancement is given as follows: ${ }^{7}$

$$
\kappa \simeq \frac{N_{T}-2}{N_{T}-2-N_{c}},
$$

where $N_{c}$ is the number of interfering signals to be rejected. The observation frame with dimension $N_{T}=M_{R}\left(Q L+L_{\Delta}\right)$ (see (10)) spans $\left(Q L+L_{\Delta}\right) / L_{R}$ segments of the targeted code with SF $L_{R}$. Due to asynchronism and multipath propagation, additional symbols will contribute at the edges. Assuming that the delay spread is insignificant, it follows that the number of constraints in (22) is $N_{c} \simeq\left\lceil\left(Q L+L_{\Delta}\right) / L_{R}\right\rceil+1$.

Using (22) and the probabilistic distribution of interference (see Figure 6), we can identify a solution that optimizes the trade-off between noise enhancement and interference reduction. Table 3 lists the relative reduction of interference and noise enhancement for different strategies. The first row

\footnotetext{
${ }^{7}$ If we strive to reject a subspace with dimension $N_{c}$ contained within the total dimension $N_{T}$, a fraction of the desired signal energy is rejected as well. It is reasonable to assume that this fraction is approximately $\left(N_{T}-\right.$ $\left.N_{c}\right) / N_{T}$. Therefore, the noise compared to the desired signal is enhanced by $N_{T} /\left(N_{T}-N_{c}\right)$. A more accurate development of (22) will be shown in a later contribution.
} 
TABLE 3: Choosing the best strategy.

\begin{tabular}{|c|c|c|c|c|c|c|c|c|}
\hline $\begin{array}{l}\text { Number of interferers to reject } \\
\text { (in-cell/neighbor } 1 / \text { neighbor } 2 \text { ) }\end{array}$ & $1 / 0 / 0$ & $2 / 0 / 0$ & $3 / 0 / 0$ & $3 / 1 / 0$ & $4 / 1 / 0$ & $4 / 2 / 0$ & $4 / 2 / 1$ & $4 / 3 / 1$ \\
\hline Interference reduction $(\mathrm{dB})$ & 1.53 & 2.64 & 3.47 & 4.41 & 5.11 & 5.83 & 6.42 & 6.98 \\
\hline Noise enhancement $(\mathrm{dB})$ & 0.32 & 0.66 & 1.04 & 1.46 & 1.91 & 2.43 & 3.01 & 3.67 \\
\hline Net gain $(\mathrm{dB})$ & 1.20 & 1.97 & 2.43 & 2.95 & 3.19 & 3.40 & 3.41 & 3.31 \\
\hline
\end{tabular}

TABLE 4: Complexity estimates of ISR variants in Mops.

\begin{tabular}{|c|c|c|c|c|c|c|}
\hline Task & DLISR-H-FC & DLISR-H-BC & DLISR-R-SD & PIC-SD & MRC & Comment \\
\hline STAR & 300 & 300 & 300 & 300 & 300 & 100 Mops per channel [37] \\
\hline Reconstruct, $\underline{\hat{Y}}_{n Q+k}^{(u, v)}$ & 259 & 363 & 363 & 311 & 0 & - \\
\hline $\mathbf{C}^{H} \underline{Y}_{n}$ & 61 & 61 & 31 & 0 & 0 & $\begin{array}{l}\text { C has higher dimension for } H \text {-variants } \\
\text { but it is sparse }\end{array}$ \\
\hline $\mathbf{Q}=\mathbf{C}^{H} \mathbf{C}$ & 246 & 246 & 61 & 0 & 0 & - \\
\hline $\mathbf{Q}^{-1} \mathbf{C}^{H} \underline{Y}_{n}$ & 282 & 282 & 1 & 0 & 0 & - \\
\hline Margin & $40 \%$ & $40 \%$ & $40 \%$ & $40 \%$ & $40 \%$ & - \\
\hline Total Mops at RSF $=8$ & 1607 & 1753 & 1058 & 855 & 420 & Appropriate when DACCA is employed \\
\hline Total Mops at RSF $=16$ & 4185 & 4476 & 1800 & 1218 & 420 & - \\
\hline Total Mops at RSF $=32$ & 13728 & 14308 & 3828 & 1944 & 420 & Appropriate when DACCA is absent \\
\hline
\end{tabular}

identifies the interferers rejected, for example, 2/1/0 means the two strongest in-cell virtual users plus the strongest outcell user of the neighbor cell with the strongest pilot channel. In the second row, the noise enhancement is computed according to (22). The net gain peaks at $3.41 \mathrm{~dB}$ suggesting that the best strategy is to reject 4 in-cell virtual users, 2 virtual users from the strongest neighbor, and one virtual user from the second strongest neighbor. In reality, the strategy (which is fixed) should be selected according to the highest load during busy hour. This ensures optimal performance at peak load and always satisfactory performance at lower loads.

\subsubsection{DLISR-H-BC}

In the DLISR-H-BC variant, we estimate the power in the virtual subspace of the serving cell and all cells in the neighbor list. The power is estimated subject to the RSF which may represent many virtual users. The best constraints are computed along the same lines as in Table 3 , but the interference reduction is based on the estimated power and not the statistical mean. This version hence adapts easily to fast fading and will attempt to reject interference most efficiently. This strategy therefore ensures that we always follow an optimal rejection strategy, provided that the powers are estimated properly and the update is done frequently.

DLISR-H-BC is more complex than DLISR-H-FC because it needs to probe the interference subspace and has to decide which constraints to reject for best performance. It can, however, work in the absence of DACCA although DACCA simplifies probing. In the absence of DACCA, interference is not generally concentrated at a low SF virtual code; it may therefore be necessary to probe the OVSF tree at higher RSF levels. This increases complexity and reduces the accuracy of probing because a few strong sources can be estimated more reliably than many weak sources.

\subsubsection{DLISR-R-SD}

In this variant, we reconstruct the virtual users using soft decision. Working at a low RSF, the $N_{v}$ OVSF virtual codes which contain most power are selected. These codes are reconstructed as virtual users' signals, and soft decision estimates based on MRC estimation are used. Note that hard decision FB is not usually an option on the DL and the fact that one virtual code is the contribution of many physical interfering users makes hard decision even more complicated.

\subsubsection{PIC-SD}

As a benchmark, we consider the PIC $[16,17]$ with SD $\mathrm{FB}$, and denote it by PIC-SD. We follow the same steps as for DLISR-R-SD; but the reconstructed interference is subtracted instead of nulled. Obviously, PIC-SD, like DLISR, takes advantage of both VIR and DACCA to improve performance and lower complexity.

\subsection{Computational complexity of DLISR}

We provide complexity estimates in Table 4 assuming VIR with an RSF of 8 for all DLISR variants, PIC-SD, and MRC. We have also listed results for an RSF of 16 and 32, respectively. We have detailed the most demanding tasks and a margin of $40 \%$ has been added to account for all other operations not listed. We assume that RSF/2 virtual codes are rejected and that three cells are actively monitored. Complexity is specified in Mops, where one operation is defined as a complex multiply-add. The numbers are appropriate for $M_{R}=1$. Roughly speaking, complexity is invariant to the SF of the desired user, and grows linearly with the number of receiving antennas. The results for $\mathrm{RSF}=8$ relate to the situation where DACCA is employed (as in our later simulations). When DACCA is not employed, an RSF of 8 is too low. We simulated the leftmost and random code-allocation 
schemes, for which details are omitted for lack of space, and found that an RSF of about 32 must be employed if the leftmost strategy is used instead of DACCA, and even higher RSF must be employed if random code allocation is employed.

The complexity of the matrix inversion is very modest. For the R-variant, it is negligible because the dimension is only 4 (with RSF $=8$ ). H-variants have higher complexities associated with the inversion but, although not evident, there are huge savings because $\mathbf{Q}$ is band diagonal as a result of VIR (low RSF approach). ${ }^{8}$ PIC-SD does not require matrix inversion and therefore has a complexity advantage over DLISR which, however, is vanishing for low RSFs.

When VIR and DACCA are employed, the complexity of our solution is moderate. Our MUD solutions require from about 1.1 to 1.7 Gops. Today's high-end signal processors offer speeds of more than 10 Gops. A requirement of 1.11.7 Gops is therefore reasonable for a mobile terminal application where cost and power consumption must be kept low. The feasibility becomes even more evident when compared with SUD (STAR-MRC); our solution requires only about 2.5-4 times the complexity of SUD. Note that our SUD candidate, STAR [22] with MRC, is comparable in complexity to the RAKE [37], which is used in current implementations. DLISR-R-SD is less complex than the H-variants but the difference is only about $50 \%$ which is considered unimportant.

If DACCA is not employed, VIR is still applicable (and should be used!) but it must target a higher RSF. This exacts a significant complexity increase of about 4-8 times ${ }^{9}$ when comparing at RSF $=32$ which as argued is a good choice when DACCA is not employed. The complexity of the $R$-variants is now four times less than the $H$-variants. It is therefore in much favor of DLISR-R-SD when higher RSFs are used.

\section{RADIO-NETWORK SIMULATOR}

The purpose of the RNS is to provide a realistic picture of the distribution of the users and how they interfere with each other. This information is then used for the link-level simulations.

The RNS starts by uniformly populating users in a homogeneous cell grid which we name the test network. Using propagation estimates, it iteratively blocks users either due to coverage or interference limitations. Once the network arrives at a stable condition, the RNS outputs the realized interference. A stable condition is characterized as one where all users can achieve the required SINR without being blocked (i.e., without exceeding the maximum power offered by the base station cell). First, we provide a mathematical formulation in Section 4.1. Then we outline the algorithm in Section 4.2.

\footnotetext{
${ }^{8}$ When the columns of $\mathbf{C}$ are arranged appropriately. Note that ISR is invariant to the arrangement of the columns of $\mathbf{C}$.

${ }^{9}$ Values are in the high end. We feel confident that many computational tricks can be exploited to reduce the complexity of reconstruction, and so forth.
}

\subsection{Network-level signal model}

The mobile unit always strives to achieve a certain SINR which is sufficient to provide a certain QoS. If the serving cell is not able to supply the power required by a mobile, the mobile is blocked. Below we define the link budget which is useful to assess the SINR at the target mobile subject to transmitted power, propagation loss, interference, and so forth. First, we briefly discuss the propagation model which is essential to the later considerations.

\subsubsection{Propagation model}

We consider the following simplified form of the OkumuraHata propagation model [38, 39]:

$$
\begin{aligned}
& L_{\mathrm{PATH}}\left(\bar{u}, \bar{v}_{u}\right) \\
& =L_{0}+10 K_{P} \log _{10}\left(\frac{\max \left\{\operatorname{dist}\left(\bar{u}, \bar{v}_{u}\right), d_{0}\right\}}{d_{0}}\right)+\Gamma_{\mathrm{LNF}},
\end{aligned}
$$

where $K_{P}$ is the propagation exponent (typically 3.5-4 for urban environments), $L_{0}$ is an offset which relates to the morphology, $\bar{u}=1, \ldots, N_{U}$ is the user index where $N_{U}$ is the total number of users in the network attempting a call, $\bar{v}=1, \ldots, \bar{N}_{\text {CELLS }}$ is the cell index where $\bar{N}_{\text {CELLS }}$ is the total number of cells, and $\operatorname{dist}(\bar{u}, \bar{v})$ is the distance between the mobile and the cell. Finally, $\Gamma_{\mathrm{LNF}}$ models the log-normal fading (LNF) and is assumed to be a normally distributed random variable, that is, $\Gamma_{\mathrm{LNF}} \in N\left\{0, \sigma_{\mathrm{LNF}}^{2}\right\}$. Note that the variables $\bar{u}, \bar{v}$, and $\bar{N}_{\text {CeLLS }}$ by definition are different from $u$, $v$, and $N_{\text {CELLS }}$ first introduced in Section 2.1. ${ }^{10}$ Considering that signals arriving from the same spatial direction will experience similar LNF, we introduce the following locationdependent modeling of the LNF:

$$
\Gamma_{\mathrm{LNF}}=X_{\mathrm{LNF}} \cos (\Theta)+Y_{\mathrm{LNF}} \sin (\Theta)
$$

where $\Theta$ is the angle between the mobile and the cell, and where $X_{\mathrm{LNF}}, Y_{\mathrm{LNF}}$ are independent zero-mean Gaussian distributed random variables with variance $\sigma_{\mathrm{LNF}}^{2}$.

\subsubsection{Generic multicell multiuser link budgets}

We define the set $g_{B}$ which contains the indices of all mobiles which are blocked. If the mobile $(\bar{u}, \bar{v})$ is not blocked (i.e., $\left.(\bar{u}, \bar{v}) \notin g_{B}\right)$, we have

$$
\begin{aligned}
S(\bar{u}, \bar{v})= & P_{\mathrm{OUT}}(\bar{u}, \bar{v})+G_{T}(\bar{u}, \bar{v}) \\
& +G_{R}-\Delta_{\mathrm{MARG}}(\bar{u}, \bar{v})-L_{\mathrm{PATH}}(\bar{u}, \bar{v}) \Longleftrightarrow \\
P_{\mathrm{OUT}}(\bar{u}, \bar{v})= & S(\bar{u}, \bar{v})-G_{T}(\bar{u}, \bar{v})-G_{R}+\Delta_{\mathrm{MARG}}(\bar{u}, \bar{v}) \\
& +L_{\mathrm{PATH}}(\bar{u}, \bar{v}),
\end{aligned}
$$

where $S$ is the signal strength at the input of the receiving

\footnotetext{
${ }^{10}$ For instance, $(u, v)$ is the $u$ th user connected to the $v$ th cell. However, $\bar{u}$ means the $\bar{u}$ th user in the network (possibly blocked).
} 
antenna, ${ }^{11} P_{\text {OUT }}$ is the power fed to the transmitting antenna, ${ }^{12} G_{T}$ and $G_{R}$ are the gains of the transmitting and receiving antennas, respectively, $\Delta_{\mathrm{MARG}}$ accounts for additional engineering margins (e.g., $P C$ margin), and $L_{\mathrm{PATH}}$ is the path loss between the serving cell and the user equipment defined in (23). We assume that the mobile antenna gain is independent of its location and $G_{R}$ is therefore location independent.

Let $\gamma_{\mathrm{REQ}}$ specify the required SINR in $\mathrm{dB}$ for a specified QoS. We assume that the required target value is the same for all mobiles. ${ }^{13}$ Assume that $\bar{v}_{u}$ is the serving cell of the $\bar{u}$ th mobile; then the required signal power at the input to the receiver, say $S_{\mathrm{REQ}}\left(\bar{u}, \bar{v}_{u}\right)$, is given in $\mathrm{dB}$ as follows:

$$
\begin{aligned}
S_{\mathrm{REQ}}\left(\bar{u}, \bar{v}_{u}\right) & =\gamma_{\mathrm{REQ}}+10 \log _{10}\left(\mathcal{N}^{\#}+\ell^{\#}(\bar{u})\right) \\
& =\gamma_{\mathrm{REQ}}+\mathcal{N}+10 \log _{10}\left(1+\frac{\ell^{\#}(\bar{u})}{\mathcal{N}^{\#}}\right),
\end{aligned}
$$

where $\mathcal{N}$ is the user-independent thermal noise power, and $\ell(\bar{u})$ is the total MAI received at the mobile $\bar{u}$ to be defined in (29). We use the \# sign to differentiate a physical value from its $\mathrm{dB}$ equivalent. We next combine (26) and (27) to find the required transmitted power:

$$
\begin{aligned}
P_{\mathrm{REQ}}\left(\bar{u}, \bar{v}_{u}\right)= & \gamma_{\mathrm{REQ}}+\mathcal{N}+10 \log _{10}\left(1+\frac{\ell^{\#}(\bar{u})}{\mathcal{N}^{\#}}\right) \\
& -G_{T}\left(\bar{u}, \bar{v}_{u}\right)-G_{R}+\Delta_{\mathrm{MARG}}+L_{\mathrm{PATH}}\left(\bar{u}, \bar{v}_{u}\right)
\end{aligned}
$$

and can now define the received interference as follows:

$$
\begin{aligned}
\ell(\bar{u})= & 10 \log _{10}\left(\sum_{\bar{u}^{\prime} \neq \bar{u}} 10^{\left(P_{\mathrm{REQ}}\left(\bar{u}^{\prime}, \bar{v}_{u}\right)-K_{\mathrm{ORTH}}-L_{\mathrm{LOSS}}\left(\bar{u}^{\prime}, \bar{v}_{u}\right)\right) / 10}\right. \\
& \left.+\sum_{\bar{v}^{\prime} \neq \bar{v}_{u}} \sum_{\bar{u}^{\prime}} 10^{\left(P_{\mathrm{REQ}}\left(\bar{u}^{\prime}, \bar{v}^{\prime}\right)-L_{\mathrm{LOSS}}\left(\bar{u}^{\prime}, \bar{v}_{u}^{\prime}\right)\right) / 10}\right) \\
& +10 \log _{10}(\mathrm{PG}),
\end{aligned}
$$

where $K_{\mathrm{ORTH}}$ is the orthogonality factor, a measure of the orthogonality loss due to multipath propagation (a typical value is $2 \mathrm{~dB}$ ), and $\mathrm{PG}=L M_{R}$ is the $\mathrm{PG}$.

We define the best server $\bar{v}_{u}$ of the mobile with index $\bar{u}$ as the serving cell $\bar{v}^{\prime}$ which requires the lowest output power to satisfy the SINR target:

$$
\bar{v}_{u}=\arg \min _{\bar{v}^{\prime}}\left\{P_{\mathrm{REQ}}\left(\bar{u}, \bar{v}^{\prime}\right) \mid \ell(\bar{u})=0\right\} .
$$

This definition suggests that we consider the best server as the cell with the strongest signal since the interference is assumed zero. In handover situations, this may not be true because of hand-over hysteresis, congestion, or load balancing. We also note that the best server, according to the definition

\footnotetext{
${ }^{11}$ We assume for simplicity that the reference point is the antenna connector and avoid this way to consider feeder losses and so forth which are immaterial for our purpose.

${ }^{12}$ The connector of the transmitting antenna is our reference point.

${ }^{13}$ In reality, the target value is determined by the outer-loop PC and differs, due to different channel conditions, slightly across mobiles which otherwise require the same service.
}

used, may not be the best choice because of orthogonal transmission, which implies that a weaker server may occasionally have better effective SINR. If the best server cannot supply the required power needed, the user is blocked. Blocking occurs either due to coverage blocking (excessive path loss) or interference blocking (excessive interference).

The size of the test network can be limited using wraparound to mitigate the edge effect. To implement wraparound, we place nine virtual images of the test network in all directions (south, south-east, east, and so forth). In the computations, the image of a cell which gives the strongest signal is chosen. For instance, to compute the required power in (28), we compute it for both the target cell and also for all its nine replicas; and then select the replica which gives the highest signal strength. Nondocumented simulations support the efficiency of network wrapping, which allows for the use of test networks smaller than 25 sites ( 5 by 5 grid).

\subsection{RNS algorithm}

The object of the algorithm is to locate mobiles in the network so that all nonblocked mobiles experience satisfactory SINR. The algorithm estimates this by uniformly distributing $\bar{N}_{\text {CELLS }} T_{\text {OFF }}$ users, where $T_{\text {OFF }}$ is the offered traffic. Then it blocks users until a stable solution is found. The RNS algorithm is illustrated by the flowchart in Figure 8. We identify a cell near the center of the grid as the target cell. Assume that the noise floor $\mathcal{N}$ and the maximum output power $P_{\text {MAX }}$ have been defined. Initially, the sets of blocked mobiles are empty, that is, $\varsigma_{\mathrm{CB}}=\varnothing$ (coverage) and $\varsigma_{\mathrm{IB}}=\varnothing$ (interference).

(1) Distribute $\bar{N}_{\text {CELLS }}$ cells on a map in a hexagonal grid.

(2) Randomly populate the test network with $\bar{N}_{\text {CELLS }} T_{\text {OFF }}$ users.

(3) Compute for every mobile-cell pair the power required for service (see (28)).

(4) Identify the best server for every user (see (30)).

(5) Users with $P_{\mathrm{REQ}}>P_{\mathrm{MAX}}, P_{\mathrm{MAX}}$ being the maximum output power which can be assigned to any individual user, are deemed to be coverage blocked and are added to the set $\delta_{\mathrm{CB}}$. The fraction of users blocked estimates the coverage blocking probability, that is, $\hat{\operatorname{Pr}} r_{\mathrm{CB}}=\operatorname{size}\left\{\wp_{\mathrm{CB}}\right\} /\left(\bar{N}_{\mathrm{CELLS}} T_{\mathrm{OFF}}\right)$.

(6) Compute the total received interference for all remaining users (see (29)).

(7) Compute required output power for all remaining users (see (28)).

(8) Block users which have $P_{\text {OUT }}>P_{\text {MAX }}$ and add to the set of interference blocked users $\diamond_{\mathrm{IB}}$.

(9) If all users that are not blocked can achieve the required SINR, stop, otherwise go to 7.

We note that the noise floor and the maximum output powers are chosen arbitrarily. By appropriate choices, we can target any desired coverage blocking.

$\hat{\operatorname{P}} r_{\text {IB }}=\operatorname{size}\left\{\delta_{\text {IB }}\right\} /\left(\bar{N}_{\text {CELLS }} T_{\text {OFF }}\right)$ estimates the probability of interference blocking. An estimate of the total SBR is then $\hat{\operatorname{P}} r_{B}=\hat{\operatorname{P}} r_{C B}+\hat{\operatorname{P}} r_{I B}$. 


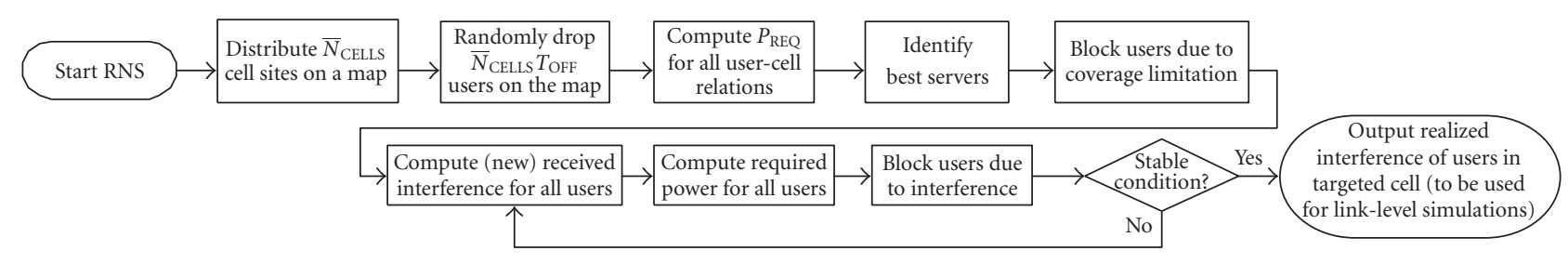

FIGURE 8: Flowchart of the radio-network simulation operations.

\section{SYSTEM-LEVEL SIMULATIONS}

\subsection{From link-level to system-level results}

The simulation model consists of the RNS (Section 4) and the link-level simulator as shown in Figure 1. The RNS provides realistic realizations of the radio-network and the linklevel simulator uses this information for BER assessments. We note that a network realization from RNS will result in the same target SINR for all mobiles; however, it is the distribution of the interference which is particularly important on the DL. For a given average offered Erlang traffic, the actual carried traffic is determined by the radio-network SBR (coverage+interference blocking). The SBR is related to the average SINR of the mobiles. This is illustrated in Figure 9 which depicts SBR as a function of the required SINR. The coverage blocking was fixed at $10 \%$ by adjusting the maximal output power $P_{\text {MAX }}$. Each SBR estimate is based on 37500 observations with the conditions otherwise stated in Section 5.2.1. Each curve corresponds to the offered traffic level specified in the legend. For a given carried traffic and SBR, the SINR from these curves dictates the PC target SINR which must be used by the link-level simulator. For instance, if we target an SBR of $20 \%$ and 4 Erlangs of traffic, the SINR target is $4.5 \mathrm{~dB}$.

\subsection{Simulation setup}

\subsubsection{RNS simulations setup}

We have considered a homogeneous hexagonal grid of 5 by 5 sites. Wrapping has been used to mitigate the edge effect. The sites have 3 sectors with pointing directions of $0^{\circ}, 120^{\circ}$, and $240^{\circ}$ azimuth, respectively. The antennas are $20 \mathrm{~m}$ high and the site-to-site distance is $250 \sqrt{5} \mathrm{~m}$. We use the verti$\mathrm{cal} /$ horizontal antenna patterns of Kathrein Werke KG, type number $742212,1950 \mathrm{MHz}$ antennas with $6^{\circ}$ electrical tilt. ${ }^{14}$ The orthogonality factor is assumed to be $2.2 \mathrm{~dB}$. We dedicate $10 \%$ of the average output power to the CPICH. Coverage blocking has been fixed at $10 \%$. We consider high data rates herein. Therefore, the coverage blocking is chosen moderately high. Table 5 summarizes the settings otherwise used.

\subsubsection{Link-level simulations setup}

In the link-level simulations, we attempted to approximate the specifications for WCDMA [2]. We have considered

\footnotetext{
${ }^{14}$ We tested antenna tilts in the range of $0^{\circ}$ to $8^{\circ}$ and selected $6^{\circ}$ because it provided the highest coverage degree with the choice of site-to-site distance and antenna heights.
}

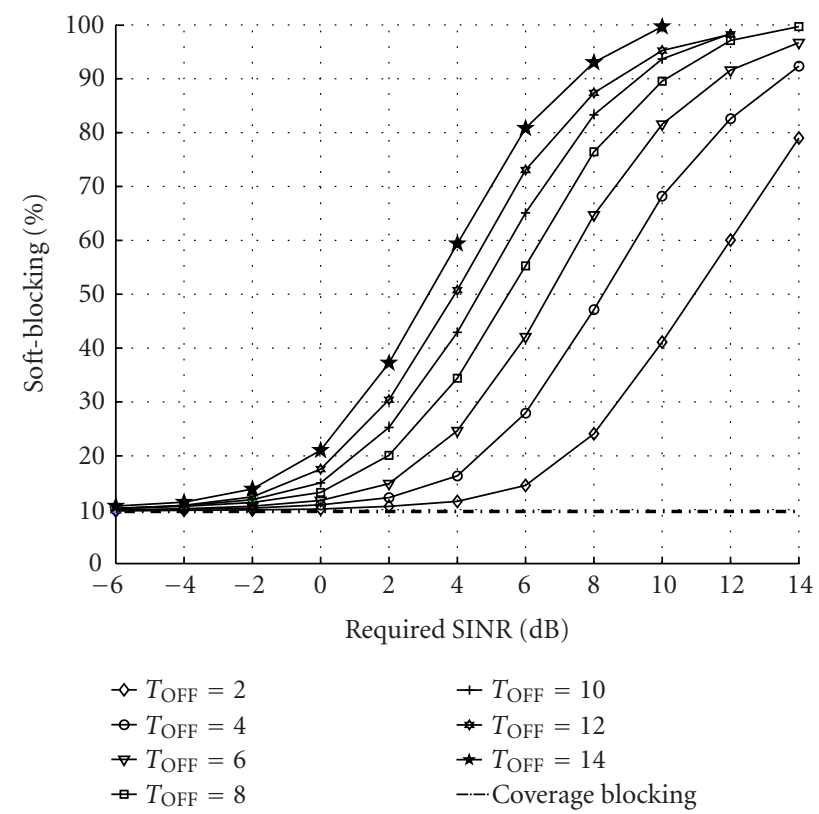

FIGURE 9: Estimated SBR as a function of the SINR target in a homogeneous system. The coverage blocking is fixed at $10 \%$ and the PG is 16.

low SF operation and high-order modulation schemes (e.g., HSPDA $[3,4])$. We use the interference realizations and pilot powers as given by the RNS as inputs to the link-level simulator. We explicitly generate signals from the serving cell and the three strongest neighbors, ${ }^{15}$ whereas the interference from the remaining cells is modeled as AWGN. In all our simulations, we consider an SF of $L=8$ which corresponds to a coded information rate of $480 \mathrm{kbps}$ with QPSK or $960 \mathrm{kbps}$ with 16 QAM when a rate-1/2 coding is assumed. The channel is Rayleigh fading [32] with chip-rate normalized Doppler $f_{D} / R_{c}$, where $R_{c}=3.86 \mathrm{Mcps}$ is the chip rate, and we consider frequency-selective fading with $P=3$ equal-strength propagation paths with random delays and interpath delays limited to 10 chips. We consider both SISO and MIMO systems. For the desired user, we implement

\footnotetext{
${ }^{15}$ Simulations with the RNS show that the desired cell and the three strongest neighbors contribute $95 \%$ of the total interference when the carried traffic is 1.6 Erlangs. This number increases with higher traffic loads.
} 
TABLE 5: Parameters used for the RNS.

\begin{tabular}{lcc}
\hline Parameter & Assumption & Comments \\
\hline Cell layout & Hexagonal grid, three-sector sites & Wrapping used to mitigate edge effect \\
Site-to-site distance & $250 \sqrt{5} \mathrm{~m}$ & \\
Antenna pattern & Kathrein 742212 with $6^{\circ}$ electrical tilt & Horizontal/vertical patterns \\
Antenna height & $20 \mathrm{~m}$ & Optimized for coverage \\
Antenna tilt & $6^{\circ}$ & $10 \%$ due to coverage, $10 \%$ due to interference \\
SBR & $20 \%$ & Relative to average cell output power \\
CPICH power & $10 \%$ & \\
Propagation model & Constant $+40 \log _{10}\left(\sqrt{x^{2}+y^{2}}\right)$ & Equal to $M_{R} L$ \\
Processing gain & 16 &
\end{tabular}

PC, ${ }^{16}$ with a PC correction factor $\Delta P_{\mathrm{PC}}$ to be updated at a rate of $1500 \mathrm{~Hz}$. The PC message is determined by comparing the estimated SINR (see Section 2.3.4) to the target SINR (coordinated with the RNS). We further impose a transmission delay of $D_{\mathrm{PC}}=1 /(1600 \mathrm{~Hz})=0.625$ millisecond and a simulated error rate on the PC bit of $\mathrm{BER}_{\mathrm{PC}}=10 \%$. Modeling closed-loop PC for all users is costly and we have therefore used a simplified model for the interfering users as illustrated in Figure 10. The signal from the unit power source is first scaled by the PC feedback to yield the transmitted power $P_{T X}^{(u, v)}$. The transmitted power is attenuated by the channel, then a Gaussian random variable with variance 0.25 is added to model practical estimation errors in the receiver. This signal is squared and used by the PC decision device to adjust the transmitted power ( $\delta_{\text {bias }}$ compensates for the bias imposed by the simulated noise), and fed back with a delay of $D_{\mathrm{PC}}$. To find the power as experienced by the target mobile, we attenuate the transmitted power by the propagation loss from the serving cell of the interferer to the desired user $L_{\mathrm{LOSS}}^{(u, v)}-L_{\mathrm{LOSS}}^{\left(d, v_{d}\right)}$, to eventually yield $\left(\psi^{(u, v)}(t)\right)^{2}$. The values of the propagation losses are obtained as a side product from the RNS.

We use STAR [22] to estimate the channels with the modifications formulated in Section 2.3.1 and Figure 7. DACCA is used with code reallocation at $75 \mathrm{~Hz}$. It is further assumed that DLISR-H-BC updates its constraints at a rate of $300 \mathrm{~Hz}$. Working at an RSF of 8 , we found that $N_{v}=2 M_{R}$ is a good rule for good performance for DLISR-R-SD in the operating

\footnotetext{
${ }^{16}$ In the absence of PC, the received power $\psi^{2}$ has a $\chi^{2}$ distribution with standard deviation $\sigma_{\psi^{2}}=1 / \sqrt{M_{T} \times M_{R}}$ that asymptotically approaches the AWGN channel at a very high diversity order $M_{T} \times M_{R} \rightarrow \infty$. With PC, however, $\psi^{2}$ has a log-normal distribution with much weaker standard deviation that quickly approaches the AWGN channel with few antenna elements only, as shown in [31]. Hence, PC significantly increases capacity and reduces the MIMO array size. Indeed, as noted in [31], if we apply the asymptotic expression for the BER in the absence of $\operatorname{PC} \operatorname{Pr}[\hat{b} \neq b]=\left(E_{b} / N_{0}\right)^{-1 / M_{T} \times M_{R}}=$ $\left(E_{b} / N_{0}\right)^{-1 / \sigma_{\psi^{2}}^{2}}$ to the case of active PC (as an approximation), we may expect to obtain (from standard deviation measurements) the same capacity with $\mathrm{PC}$ and $3 \times 2$ antennas as would be obtained without $\mathrm{PC}$ and $30 \times 2$ antennas!
}

region of interest (about 5\% BER). The PIC-SD interestingly shows strong sensitivity to this parameter and the best choice proves to be $N_{v}=M_{R}$. The parameters most commonly utilized in the simulations, unless otherwise specified, are summarized in Table 6. All BER estimates reported are derived from at least 150 RNS realizations and each realization was run for at least 19000 symbols.

\subsection{SISO with QPSK modulation}

We consider first a SISO system with QPSK modulation. The SBR is $20 \%$ and the SF is $L=8$. We employ one channelization-code group composed of $L=8$ orthogonal Walsh codes. Note that the high soft-blocking ratio considered reflect the high data rate services that we are considering. The carried traffic is hence hard limited to a maximum of 8 users. Code blocking occurs rarely with the traffic loads we consider and its influence is vanishing compared to the SBR of $20 \%$. This claim is true for all simulations cited herein.

Figure 11 shows the uncoded BER as a function of the carried Erlang traffic in the network. Our proposed DLISR variants significantly outperform MRC. They provide Erlang capacity gains of $3.5 \mathrm{~dB}$ (DLISR-H-BC) > $3.2 \mathrm{~dB}$ (DLISR-RSD) > $1.6 \mathrm{~dB}$ (DLISR-H-FC), ${ }^{17}$ respectively, over MRC-based SUD at 5\% BER. Although PIC-SD is similar to DLISR-R-SD, it can only offer a gain of $2.6 \mathrm{~dB}$. This illustrates the advantage of linearly constrained beamforming compared to subtraction. ${ }^{18}$ With DLISR-H-BC, we achieve the highest spectral efficiency of $0.78 \mathrm{bps} / \mathrm{Hz}$, where spectral efficiency is defined as $\eta_{S}=\log _{2}\left(M_{\text {Mod }}\right) T_{\text {Erl }} / L$, where $M_{\text {Mod }}$ denotes the number of symbols in the signal constellation, and $T_{\mathrm{Erl}}$ is the carried Erlang traffic (at 5\% BER). It is noteworthy that the $\mathrm{H}$ mode on the UL did not demonstrate as good performance. The pronounced near-far situations on the DL makes its application attractive.

\footnotetext{
${ }^{17}$ We use for simplicity ">" to say that the gain is "greater than."

${ }^{18}$ Note the similarity of these two: with PIC, interference is reconstructed and subtracted; with DLISR-R-SD, the reconstructed interference is nulled.
} 


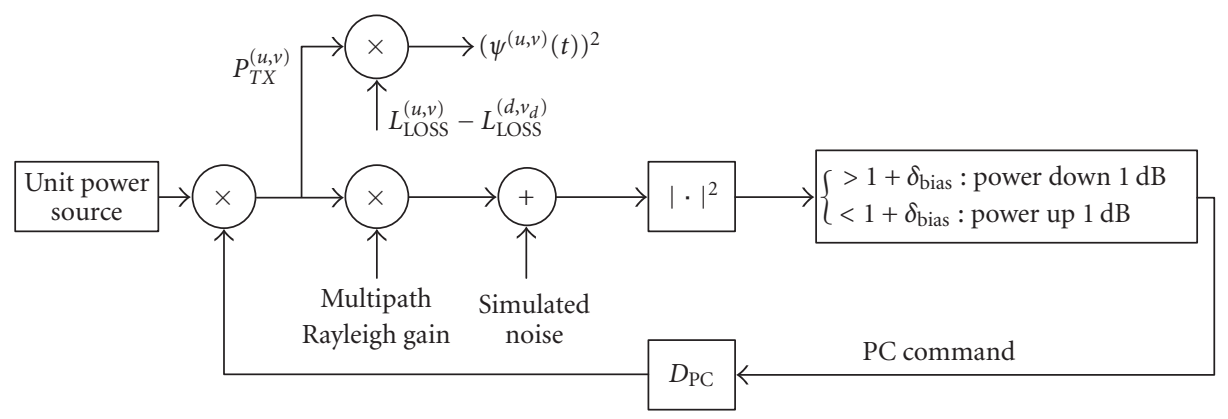

Figure 10: Simplified PC modeling used to model PC (for interfering users only).

TABle 6: Parameters used in link-level simulations (unless otherwise specified).

\begin{tabular}{ccl}
\hline Parameter & Value & Comment \\
\hline$R_{c}$ & $3.84 \mathrm{Mcps}$ & Chip rate \\
$P$ & $3(0 \mathrm{~dB}, 0 \mathrm{~dB}, 0 \mathrm{~dB})$ & Number of paths (relative average strength) \\
$f_{c}$ & $1.9 \mathrm{GHz}$ & Carrier frequency \\
$f_{e}$ & $0 \mathrm{~Hz}$ & Frequency error (we assume that frequency offset \\
$f_{D}$ & $8.9 \mathrm{~Hz}$ & errors have been compensated, see [40]) \\
$L$ & 8 & Doppler frequency (i.e., $5 \mathrm{Kmph})$ \\
$f_{\mathrm{PC}}$ & $1600 \mathrm{~Hz}$ & SF \\
$\Delta P_{\mathrm{PC}}$ & $\pm 1 \mathrm{~dB}$ & Frequency of PC updating \\
$\mathrm{BER}$ & $10 \%$ & PC adjustment \\
$\delta \tau$ & $2 \mathrm{ppm}$ & Simulated PC BER \\
$\delta t$ & $10 \mathrm{chips}$ & Symbol clock drift (linear) \\
$\Delta \tau$ & $75 \mathrm{~Hz}$ & Maximal delay spread \\
$f_{\mathrm{DACCA}}$ & & DACCA reassignment rate \\
\hline
\end{tabular}

Note that if we instead compare capacities at BER levels below $3 \mathrm{~dB}$ and above 5\%, respectively (i.e., $2.5 \%$ and $10 \%$ ), the DLISR-H-BC capacity gains over MRC are $4.5 \mathrm{~dB}$ and $2.3 \mathrm{~dB}$, respectively. It is therefore advantageous for DLISR (and MUD solutions in general) to compare along lower BER levels. Our internal studies have shown that 5\% is an appropriate target if a rate- $1 / 2$ convolutional code with constraint length 9 is assumed. We therefore continue to aim at $5 \%$.

\section{4. $2 \times 2$ MIMO with QPSK modulation}

We now consider a $2 \times 2 \mathrm{MIMO}$ system. The SF is still 8 but the PG is 16 because of the extra antenna. Since we have two transmitting antennas, we have defined two groups of channelization codes. One group consists of $L=8$ orthogonal Walsh codes; the second group likewise consists of 8 orthogonal codes obtained from the first group by $45^{\circ}$ rotation (see the example in Section 2.1). Results are shown in Figure 12.

DLISR-H-BC, DLISR-R-SD, PIC-SD, and MRC achieve the same relative capacity gain of about $3.9 \mathrm{~dB}$ compared to SISO. The advantage of linearly constrained beamforming (DLISR-R-SD) compared to subtraction (PIC-SD) is confirmed in this situation as well. The best spectral efficiency of $1.95 \mathrm{bps} / \mathrm{Hz}$ is again achieved by DLISR-H-BC. It is obvious that about $3 \mathrm{~dB}$ of these gains are due to the antenna gain. The rest is a combination of diversity and statistical multi- plexing gain on the air interface. DLISR-H-FC improves in MIMO compared to SISO achieving a relative gain of $5.1 \mathrm{~dB}$ compared to SISO. DLISR-H-FC experiences a statistical gain because more users are active in the MIMO system and randomness hence plays a less dominant role. Since this variant uses completely fixed constraints, interference energy is more likely to be concentrated where expected.

\section{5. $4 \times 4$ MIMO with QPSK modulation}

We increase the number of receive and transmit antennas to four. Four code groups were determined by computer simulations where the objective was to minimize the intergroup cross-correlation. Results are shown in Figure 13 (for MRC, ISRDL-H-BC, and PIC-SD). The spectral efficiency of both DLISR and MRC doubles, compared to the $2 \times 2$ MIMO system. We are hence able to retain our MUD advantage of at least $3 \mathrm{~dB}$ over MRC-based SUD. PIC-SD as usual performs worse than DLISR and can only provide a gain of $2.1 \mathrm{~dB}$ over MRC. With DLISR-H-BC, we can now support 17 Erlangs of $480 \mathrm{kbps}$ traffic per sector corresponding to a spectral efficiency of 4 bits/Hz/sector. Comparing SISO, $2 \times 2 \mathrm{MIMO}$, and $4 \times 4 \mathrm{MIMO}$, we notice that capacity increases linearly with the number of antennas. This linear relationship was also found by [9] for the MMSE MUD in an interference-limited cellular system. In cellular interference-limited systems, the 


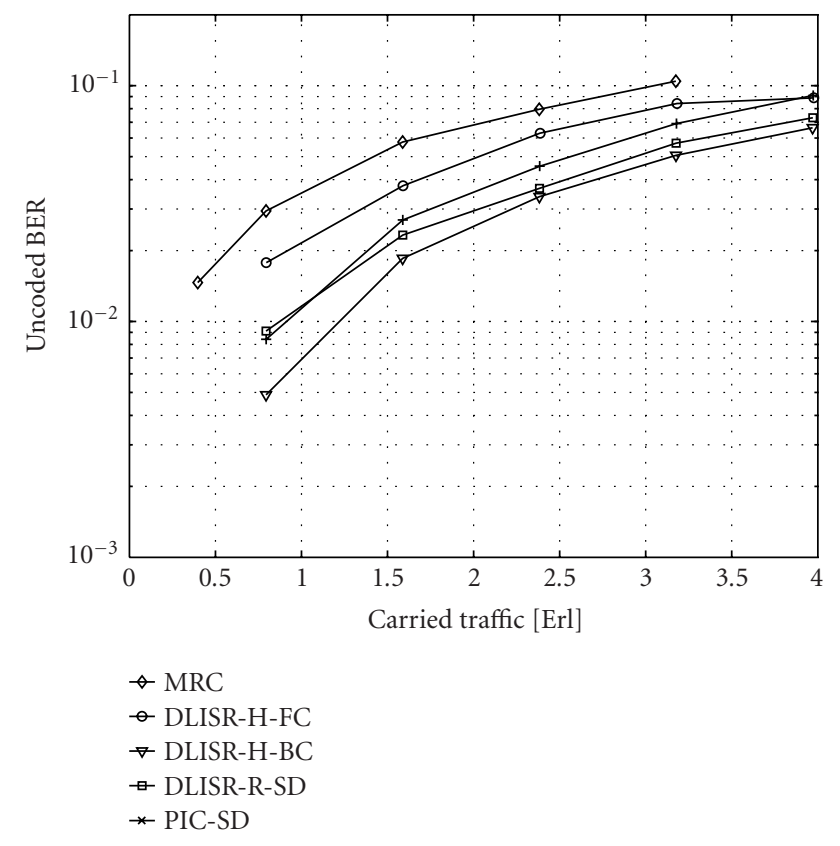

FIGURE 11: Uncoded BER performance as a function of the offered traffic. The modulation is QPSK and the channel is SISO. The SF is 8 corresponding to an information rate of $480 \mathrm{kbps}$ (rate-1/2 coding assumed).

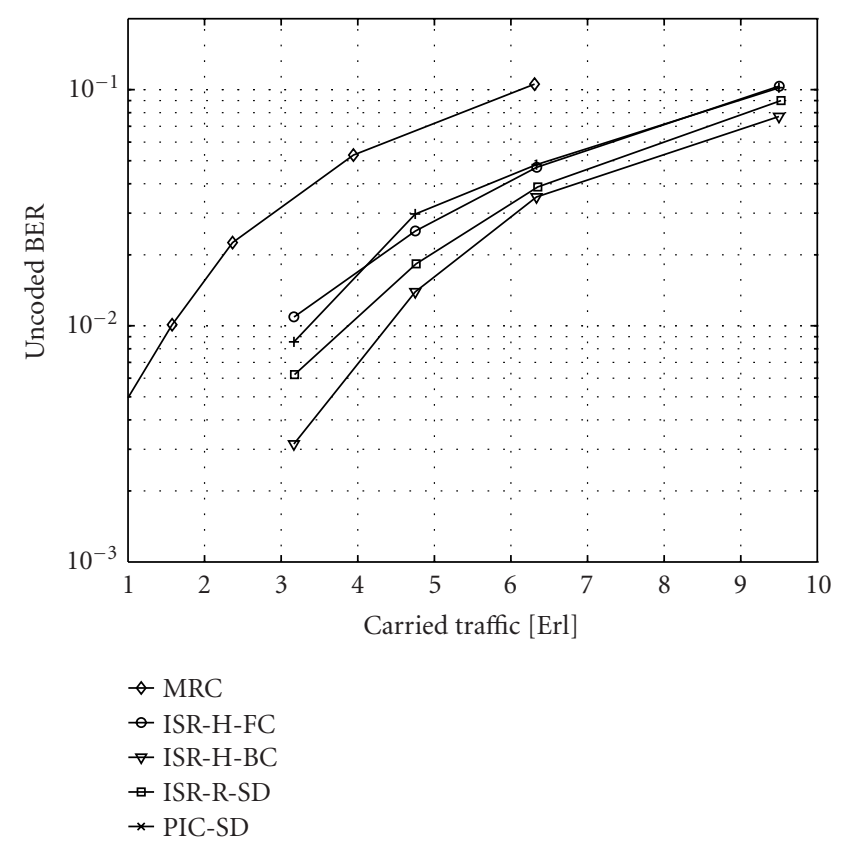

FIGURE 12: Uncoded BER performance as a function of the offered traffic. The modulation is QPSK and the channel is $2 \times 2$ MIMO. The SF is 8 corresponding to an information rate of $480 \mathrm{kbps}$ (rate$1 / 2$ coding assumed).

gain is limited to the antenna gain and is therefore dictated by the number of receive antennas. Note that multiple transmit

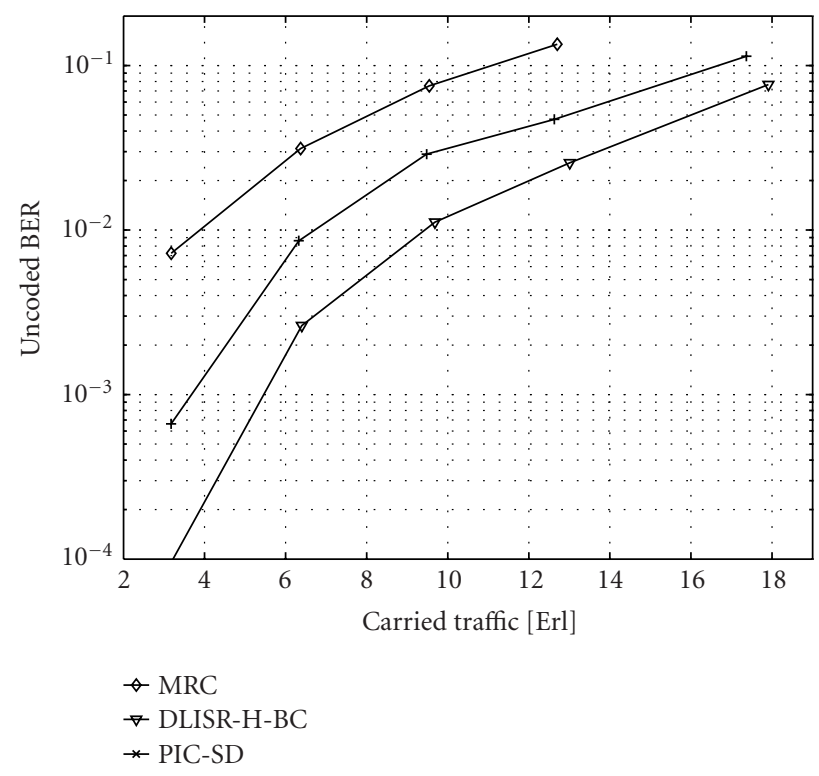

FIGURE 13: Uncoded BER performance as a function of the offered traffic. The modulation is QPSK, the channel is $4 \times 4 \mathrm{MIMO}$, and the SBR is $20 \%$. The SF is 8 corresponding to an information rate of $480 \mathrm{kbps}$ (rate-1/2 coding assumed).

antennas still serve to alleviate the shortage of OVSF codes and can provide additional time diversity.

\section{6. $2 \times 2$ MIMO with 16-QAM modulation}

We use the same settings as in Section 5.4 but consider now 16-QAM modulation corresponding to a bit rate of $960 \mathrm{kbps}$ after rate-1/2 coding. Figure $14 \mathrm{a}$ shows the uncoded BER as a function of the carried traffic. We have used the 16-QAM symbol constellation suggested in [4].

The capacity gain of DLISR compared to MRC becomes dominant offering $8.1 \mathrm{~dB}$ capacity increase achieved with DLISR-H-BC. DLISR-R-SD performs slightly worse with $7.7 \mathrm{~dB}$ gain over MRC, but as usual, outperforming PICSD which only provides a gain of $6.7 \mathrm{~dB}$. The remarkable gains over MRC are a result of increased data rate which effectively exacerbates the near-far situations because interference is limited to fewer sources. Compared to the QPSK results, the carried Erlang traffic is reduced by about $5.4 \mathrm{~dB}$ for DLISR variants. The spectral efficiency, which decreases less due to the doubled symbol rate, is $1.1 \mathrm{bps} / \mathrm{Hz}$ for DLISR$\mathrm{H}-\mathrm{BC}$ corresponding to a reduction of $2.6 \mathrm{~dB}$ compared to MIMO QPSK.

Higher capacities can always be achieved at the expense of increased SBR because it implies higher SINR operating point, even though the carried traffic is constant. To see the effect, Figure $14 \mathrm{~b}$ shows performance with SBR $=60 \%$. The spectral efficiency is increased for all modes. For instance, the DLISR-H-BC spectral efficiency is increased by $1.8 \mathrm{~dB}$, yielding an absolute spectral efficiency of about $1.5 \mathrm{bps} / \mathrm{Hz}$. This illustrates the important trade-off between capacity and 


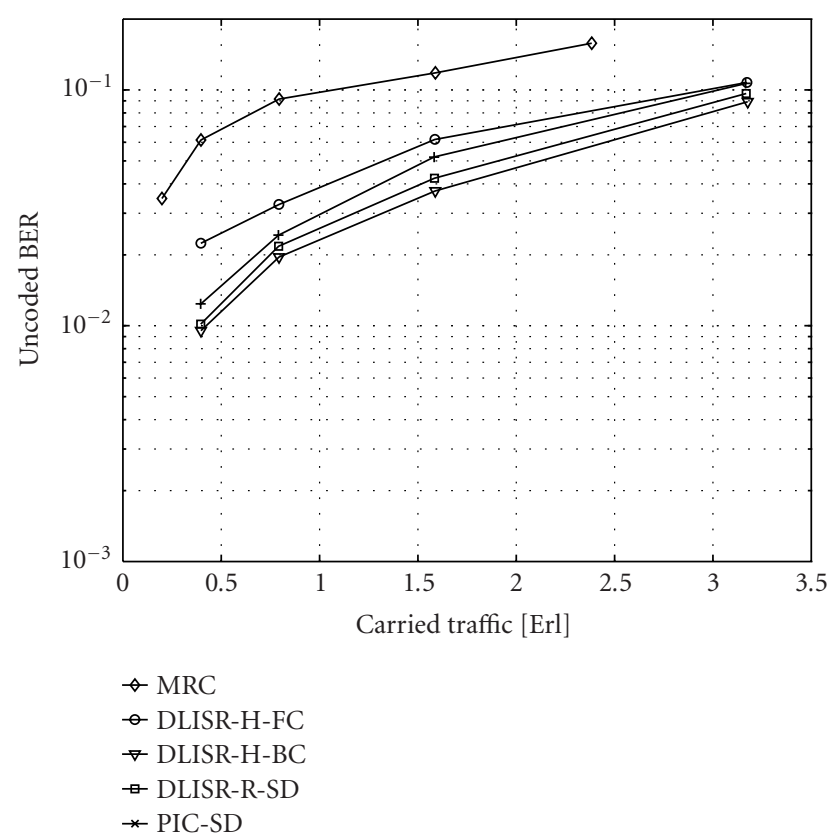

(a)

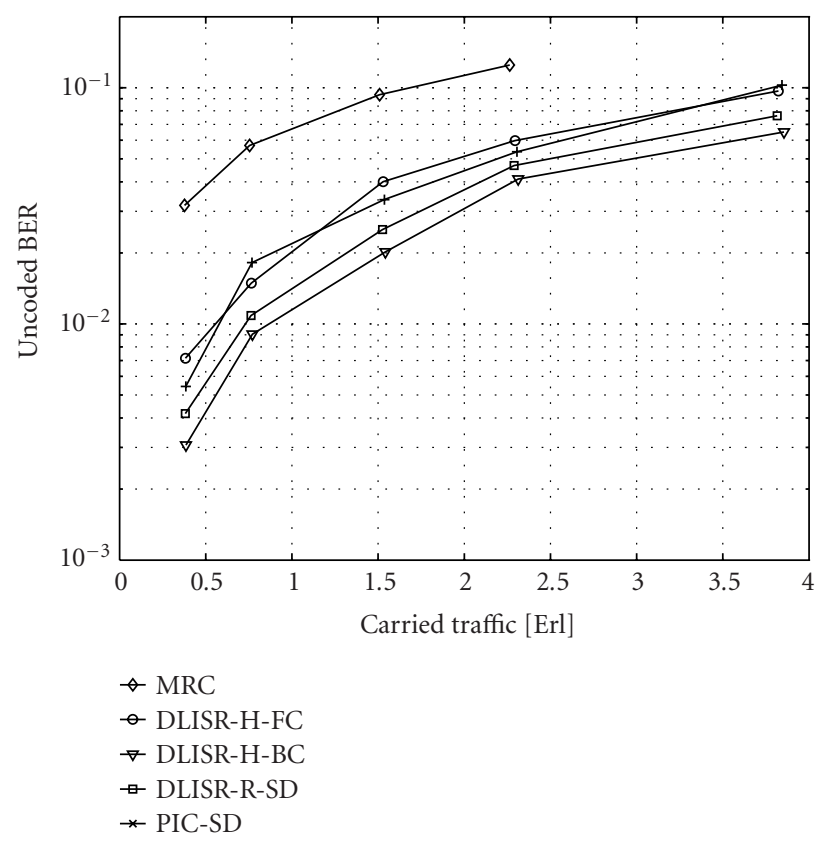

(b)

FIGURE 14: Uncoded BER performance as a function of the offered traffic. The modulation is 16 QAM, the channel is $2 \times 2 \mathrm{MIMO}$, and the SF is 8 corresponding to an information rate of $960 \mathrm{kbps}$ (rate-1/2 coding assumed). (a) The SBR is $20 \%$. (b) The SBR is $60 \%$.

network SBR. Higher SBR reduces the benefit of DLISR compared to MRC slightly, but it is still a significant $6.5 \mathrm{~dB}$ with DLISR-H-BC. MRC benefits more from increased SBR because in-cell interference becomes dominant and therefore an orthogonality gain, which is more pronounced for MRC, is achieved.

\section{CONCLUSION}

In this paper, we have presented a new MUD for DL MIMO systems. Our solution is based on previously presented ISR and is denoted DLISR. We have defined three variants of DLISR with different performances and complexities. The DLISR variants share one common feature, they employ VIR and they can benefit from dynamic allocation of channelization codes at the base station using the DACCA technique. VIR significantly reduces complexity because interference is rejected at a low (virtual) SF. With DACCA, the base station assigns channelization codes with the aim of concentrating interference in a small portion of the OVSF code tree. With DACCA, VIR therefore becomes even more efficient because we can attack interference at a lower SF hence reducing complexity further. We note that only one of our solutions requires DACCA. The remaining solutions benefit from DACCA in terms of complexity.

Performance of DLISR has been evaluated with the aid of a realistic simulation model consisting of an RNS and a link-level simulator. The RNS generates interference scenar- ios similar to those experienced in real life. These realizations of interference are used by the link-level simulator to produce BER performance statistics. At both levels, we have strived to use realistic assumptions. As a benchmark, we have considered the MRC-based SUD and the PIC-SD.

The Erlang capacity of the network is found to grow linearly with the number of receive antennas for both MRCbased SUD and our new DLISR MUD despite the existence of interference. Significant increases of capacity are achieved with DLISR which offers capacity gains over MRC-based SUD of at least $3 \mathrm{~dB}$ for QPSK (480 kbit/s) and about 6.5$8.1 \mathrm{~dB}$ when $16 \mathrm{QAM}(960 \mathrm{kbit} / \mathrm{s})$ is employed. A $4 \times 4$ MIMO system can support 17 Erlangs of $480 \mathrm{kbit} / \mathrm{s}$ traffic per sector corresponding to a spectral efficiency of 4 bits/s/Hz. DLISR-H-BC always achieves best performance outperforming DLISR-R-SD by about $0.3-0.5 \mathrm{~dB}$. DLISR-RSD outperforms PIC-SD by $0.5-0.9 \mathrm{~dB}$, hence illustrating the advantage of linearly constrained beamforming (DLISR-R$\mathrm{SD}$ ) compared to subtraction (PIC-SD). DLISR-H-FC generally achieves the least gain over MRC, but it also possesses the simplest structure.

Our DLISR solutions have low complexity when DACCA is employed in UTRAN. The gains cited herein are achieved at a complexity of about $1.6 \mathrm{Gops}$, which is only about 4 times that of SUD, and close to the complexity of PIC-SD. The realistic assumptions of our study suggest that our solution is low risk. The new DLISR MUD is therefore a serious candidate for DL MUD in CDMA-based MIMO and SISO systems. 


\section{ACKNOWLEDGMENT}

This work was supported by the Bell/Nortel/NSERC Industrial Research Chair in Personal Communications and the Natural Sciences and Engineering Research Council of Canada (NSERC) Research Grants Program.

\section{REFERENCES}

[1] F. Adachi, M. Sawahashi, and H. Suda, "Wideband DS-CDMA for next-generation mobile communication systems," IEEE Communications Magazine, vol. 36, no. 9, pp. 56-69, 1998.

[2] R. Prasad and T. Ojanper, "An overview of CDMA evolution toward wideband CDMA," IEEE Communications Surveys, vol. 1, no. 1, pp. 2-29, 1998.

[3] Third Generation Partnership Project, "Ultra high speed downlink packet access (HSDPA) (Release 5)," 3GPP Tech. Spec. (TS) 25.308, v. 5.2.0, Technical Specification Group Radio Access Network, March 2002.

[4] Third Generation Partnership Project, "Physical layer aspects of UTRA high speed downlink packet access (Release 4)," 3GPP Tech. Rep. (TR) 25.848, v. 4.0.0, Technical Specification Group Radio Access Network, March 2001.

[5] G. J. Foschini and M. J. Gans, "On limits of wireless communications in a fading environment when using multiple antennas," Wireless Personal Communications, vol. 6, no. 3, pp. 311-335, 1998.

[6] S. Verdu, Multiuser Detection, Cambridge University Press, Cambridge, UK, 1998.

[7] A. Duel-Hallen, J. Holtzman, and Z. Zvonar, "Multiuser detection for CDMA systems," IEEE Personal Communications, vol. 2, no. 2, pp. 46-58, 1995.

[8] S. Moshavi, "Multi-user detection for DS-CDMA communications," IEEE Communications Magazine, vol. 34, no. 10, pp. 124-136, 1996.

[9] S. Catreux, P. F. Driessen, and L. J. Greenstein, "Attainable throughput of an interference-limited multiple-input multiple-output (MIMO) cellular system," IEEE Trans. Communications, vol. 49, no. 8, pp. 1307-1311, 2001.

[10] S. Catreux, P. F. Driessen, and L. J. Greenstein, "Simulation results for an interference-limited multiple-input multipleoutput cellular system," IEEE Communications Letters, vol. 4, no. 11, pp. 334-336, 2000.

[11] R. Lupas and S. Verdu, "Linear multiuser detectors for synchronous code-division multiple-access channels," IEEE Transactions on Information Theory, vol. 35, no. 1, pp. 123136, 1989.

[12] R. Lupas and S. Verdu, "Near-far resistance of multiuser detectors in asynchronous channels," IEEE Trans. Communications, vol. 38, no. 4, pp. 496-508, 1990.

[13] K. S. Schneider, "Optimum detection of code division multiplexed signals," IEEE Trans. on Aerospace and Electronics Systems, vol. 15, pp. 181-185, 1979.

[14] Z. Xie, R. T. Short, and C. K. Rushforth, "A family of suboptimum detectors for coherent multiuser communications," IEEE Journal on Selected Areas in Communications, vol. 8, no. 4, pp. 683-690, 1990.

[15] U. Madhow and M. Honig, "MMSE interference suppression for direct-sequence spread-spectrum CDMA," IEEE Trans. Communications, vol. 42, no. 12, pp. 3178-3188, 1994.

[16] R. Kohno, H. Imai, M. Hatori, and S. Pasupathy, "Combination of an adaptive array antenna and a canceller of interference for direct-sequence spread-spectrum multiple-access system," IEEE Journal on Selected Areas in Communications, vol. 8, no. 4, pp. 675-682, 1990.
[17] M. K. Varanasi and B. Aazhang, "Multistage detection in asynchronous code-division multiple-access communications," IEEE Trans. Communications, vol. 38, no. 4, pp. 509519,1990

[18] P. Patel and J. Holtzman, "Analysis of a simple successive interference cancellation scheme in a DS/CDMA system," IEEE Journal on Selected Areas in Communications, vol. 12, no. 5, pp. 796-807, 1994.

[19] C. S. Wijting, T. Ojanperä, M. J. Juntti, K. Kansanen, and R. Prasad, "Groupwise serial multiuser detectors for multirate DS-CDMA," in IEEE 49th Vehicular Technology Conference, vol. 1, pp. 836-840, Houston, Tex, USA, July 1999.

[20] D. Guo, L. K. Rasmussen, S. Sun, and T. J. Lim, "A matrixalgebraic approach to linear parallel interference cancellation in CDMA," IEEE Trans. Communications, vol. 48, no. 1, pp. $152-161,2000$.

[21] L. K. Rasmussen, T. J. Lim, and A. L. Johansson, "A matrixalgebraic approach to successive interference cancellation in CDMA," IEEE Trans. Communications, vol. 48, no. 1, pp. 145151,2000

[22] S. Affes and P. Mermelstein, "A new receiver structure for asynchronous CDMA: STAR - the spatio-temporal arrayreceiver," IEEE Journal on Selected Areas in Communications, vol. 16, no. 8, pp. 1411-1422, 1998.

[23] H. Hansen, S. Affes, and P. Mermelstein, "A beamformer for CDMA with enhanced near-far resistance," in Proc. IEEE International Conference on Communications, vol. 3, pp. 15831587, Vancouver, British Columbia, Canada, June 1999.

[24] A. J. Viterbi, CDMA Principles of Spread Spectrum Communication, vol. 1 of Addison-Wesley Wireless Communication Series, Addison-Wesley, 1997.

[25] G. E. Bottomley, T. Ottosson, and Y.-P. E. Wang, "A generalized RAKE receiver for interference suppression," IEEE Journal on Selected Areas in Communications, vol. 18, no. 8, pp. 1536-1545, 2000.

[26] G. Kutz and A. Chass, "On the performance of a practical downlink CDMA generalized RAKE receiver," in IEEE 56th Vehicular Technology Conference, vol. 3, pp. 1352-1356, Vancouver, British Columbia, Canada, September 2002.

[27] G. Kutz and A. Chass, "Low complexity implementation of a downlink CDMA generalized RAKE receiver," in IEEE 56th Vehicular Technology Conference, vol. 3, pp. 1357-1361, Vancouver, British Columbia, Canada, September 2002.

[28] M. F. Madkour, S.C. Gupta, and Y.-P. E. Wang, "Successive interference cancellation algorithms for downlink W-CDMA communications," IEEE Trans. on Wireless Communications, vol. 1, no. 1, pp. 169-177, 2002.

[29] Third Generation Partnership Project, "Spreading and modulation (FDD) (Release 5)," 3GPP Tech. Spec. (TS) 25.213, v. 5.2.0, Technical Specification Group Radio Access Network, September 2002.

[30] S. Affes, H. Hansen, and P. Mermelstein, "Interference subspace rejection: a framework for multiuser detection in wideband CDMA," IEEE Journal on Selected Areas in Communications, vol. 20, no. 2, pp. 287-302, 2002.

[31] S. Affes, K. Lajnef, K. Cheikhrouhou, and P. Mermelstein, "Adaptive MIMO-diversity selection with closed-loop power control over wireless CDMA rayleigh-fading channels," in Proc. 7th IEEE International Symposium on Signal Processing and Its Applications, vol. 1, pp. 41-44, Paris, France, July 2003.

[32] W. C. Jakes Jr., Microwave Mobile Communications, John Wiley \& Sons, New York, NY, USA, 1974.

[33] T. Minn and K.-Y. Siu, "Dynamic assignment of orthogonal variable-spreading-factor codes in W-CDMA," IEEE Journal on Selected Areas in Communications, vol. 18, no. 8, pp. 14291440, 2000. 
[34] Y.-C. Tseng, C.-M. Chao, and S.-L. Wu, "Code placement and replacement strategies for wideband CDMA OVSF code tree management," in Proc. IEEE Global Telecommunications Conference, vol. 1, pp. 562-566, San Antonio, Tex, USA, November 2001.

[35] H. Hansen, S. Affes, and P. Mermelstein, "Downlink MIMO multiuser detection with interference subspace rejection," in Proc. IEEE Global Telecommunications Conference, San Francisco, Calif, USA, December 2003.

[36] S. Affes, H. Hansen, and P. Mermelstein, "Near-far resistant single-user channel identification by interference subspace rejection in wideband CDMA," in Proc. IEEE Workshop on Signal Processing Advances in Wireless Communications, pp. 5457, Taoyuan, Taiwan, March 2001.

[37] K. Cheikhrouhou, S. Affes, and P. Mermelstein, "Impact of synchronization on performance of enhanced array-receivers in wideband CDMA networks," IEEE Journal on Selected Areas in Communications, vol. 19, no. 12, pp. 2462-2476, 2001.

[38] Y. Okumura, E. Ohmori, T. Kawano, and K. Fukuda, "Field strength and its variability in VHF and UHF land-mobile radio service," Review of the Electrical Communication Laboratory, vol. 16, no. 9-10, pp. 825-873, 1968.

[39] M. Hata, "Empirical formula for propagation loss in land mobile radio services," IEEE Trans. Vehicular Technology, vol. VT29, no. 3, pp. 317-325, 1980.

[40] B. Smida, S. Affes, and P. Mermelstein, "Frequency and time synchronization for the CDMA array-receiver STAR with interference subspace rejection," in IEEE Vehicular Technology Conference, vol. 6, pp. 3290-3295, Orlando, Fla, USA, October 2003 .

Henrik Hansen was born in Copenhagen, Denmark, on April 19, 1971. In 1997, he received the M.S. degree with communication as specific field of engineering and in March 2001, he received the Ph.D. degree in electrical engineering, both from the Technical University of Denmark, Denmark. During his Ph.D. study, he was a Visiting Researcher at INRS-Telecommunications, Montréal, Quebec, Canada. The topic of the Ph.D. study was digital demodulation principles for CDMA systems, with special focus on multiuser detection. Since January 2001, he has been employed as a Radio-Network Planner at Ericsson, Denmark. He is primarily involved in the planning of WCDMA radio networks with focus on the dimensioning of the air interface. He is also active in various research projects within wireless technologies. His research interests include digital modulation/demodulation principles, multiuser detection, interference cancellation, adaptive antennas, space-time processing, coding, and radio-network planning and optimization.

Sofiène Affes received the "Diplôme d'Ingénieur" in electrical engineering in 1992, and the Ph.D. degree with honors in signal processing in 1995, both from the École Nationale Supérieure des Télécommunications, Paris, France. He has been since then with INRS-Telecommunications, University of Quebec, Montreal, Canada, as a Research Associate from 1995 till 1997, then as an Assistant Professor till

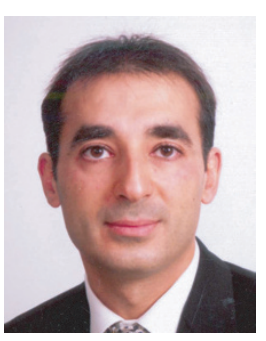
2000. Currently, he is an Associate Professor in the Personal Communications Group. His research interests are in wireless communications, statistical signal and array processing, synchronization, multiuser detection, and MIMO systems. In 1997, he participated in the major program in personal and mobile communications of the Canadian Institute for Telecommunications Research. From 1998 to 2001, he has been leading the radiodesign and signal processing activities of the Bell/Nortel/NSERC Industrial Research Chair in Personal Communications at INRSTelecommunications. Professor Affes is the Corecipient of the 2002 Prize for Research Excellence of INRS and currently holds a Canada Research Chair in High-Speed Wireless Communications.

Paul Mermelstein received the B. Eng. degree in engineering physics from McGill University, Montreal, Canada, in 1959 and the S.M., E.E., and D.S. degrees in electrical engineering from Massachusetts Institute of Technology, Cambridge, Mass, in 1960, 1963, and 1964, respectively. From 1964 to 1973, he was a member of the technical staff in the Speech and Communications Research Department, Bell Laboratories, Murray Hill, NJ, carrying out research studies in speech generation and recognition. From 1973 to 1977, he was with Haskins Laboratories, New Haven, Conn, conducting research in speech perception and recognition. He moved to Bell Northern Research, later Nortel Networks, in 1977 to lead a speech communications research group. In 1994, he moved to INRS-Telecommunications, University of Quebec, as Professor and the Bell/Nortel/NSERC Industrial Research Chair in Personal Communications. From 1994 to 2000, he was the leader of the major program in personal and mobile communications of the Canadian Institute for Telecommunications Research. He is a Fellow of the IEEE and the Acoustical Society of America and served as Editor for Speech Communications of the IEEE Transactions on Communications. 